\title{
Guidance and Control Design for a Class of Spin-Stabilized Projectiles with a Two-Dimensional Trajectory Correction Fuze
}

\author{
Yi Wang, Wei-dong Song, Dan Fang, and Qing-wei Guo \\ Electronic Engineering Department, Shijiazhuang Mechanical Engineering College, No. 97 Heping West Road, \\ Shijiazhuang, Hebei 050003, China \\ Correspondence should be addressed to Yi Wang; wangyi050926@163.com
}

Received 27 May 2015; Accepted 14 July 2015

Academic Editor: Christopher J. Damaren

Copyright (C) 2015 Yi Wang et al. This is an open access article distributed under the Creative Commons Attribution License, which permits unrestricted use, distribution, and reproduction in any medium, provided the original work is properly cited.

A guidance and control strategy for a class of 2D trajectory correction fuze with fixed canards is developed in this paper. Firstly, correction control mechanism is researched through studying the deviation motion, the key point of which is the dynamic equilibrium angle. Phase lag of swerve response is the dominating factor for correction control, and formula is deduced with the Mach number as argument. Secondly, impact point deviation prediction based on perturbation theory is proposed, and the numerical solution and application method are introduced. Finally, guidance and control strategy is developed, and simulations to validate the strategy are conducted.

\section{Introduction}

An important objective for future artillery projectiles is high delivery accuracy, in other words, improved aim to reduce round expenditure. In last few decades, several interesting concepts of guided projectiles were proposed, and they could be divided into three categories in terms of the control mechanisms employed: aerodynamic surfaces (nosemounted canards, tail fins, and wedge-shaped paddle) [19], jet thrusters (gas or explosive thrusters) [10-13], and inertial loads (translating or rotating internal masses) [14, 15]. The first category of control mechanism presents some advantages over the other two: on one hand, the aerodynamic surfaces provide continuous time correction easier than jet thrusters; on the other hand, they potentially give the designer more control authority and bear more resemblance to missile trajectory control system. Furthermore, guided projectile concepts involving aerodynamic control devices can be divided into two categories: fin-stabilized (such as the US M712 Copperhead and the M982 Excalibur) and spin-stabilized (such as the US XM 1156 Precision Guidance Kit). Spin-stabilized guided projectiles are generally equipped with a roll-decoupled trajectory correction fuze, designed to provide range and lateral trajectory correction, while at the same time they rely on the high spin rate of the aft part for airframe stability. But the high spin rate creates an important coupling between the normal and lateral axes of the body, which makes the dynamic characteristic rather complex.

Swerve response of the spin-stabilized is a key point of the dynamic characteristic to be studied, when control input is added. Ollerenshaw and Costello gave the closeform formulas for the magnitude and phase angle of a projectile excited by a control force in terms of fundamental and pointed out that spin-stabilized projectiles respond out of phase to control-force inputs forward of the center of pressure [16]. Fresconi and Plostins studied various control mechanism strategies for spin-stability projectiles and gave the correction authority under different conditions [17]. But this paper just introduced the strategies, and the conclusion could not be used in engineering application directly.

The trajectory correction fuze incorporates all the necessary electromechanical devices for guidance, navigation, and control system of the airframe. Due to the limit of the volume of the fuze, all the electromechanical devices must have high integration degree, which requires the guidance algorithm to have small amount of calculation but high accuracy. 
The five main types of guidance most considered in guided ammunition contexts are trajectory shaping, model predictive guidance, path following, impact point prediction control, and proportional navigation. When only small correction to a trajectory is possible, only the later four are applicable. In model predictive guidance a model of the projectile and the environment is used in each update instant to compute a sequence of control actions, given the current state [18-20]. The accuracy of the guidance is decided by the model of the projectile. If we want high accuracy, a complex model is needed, and the more state variables are acquired, the higher accuracy is got. The disadvantage of this method is that the amount of calculation is too large. In path following guidance, the current position of the projectile is compared with the nominal trajectory and a control is applied to bring the projectile to the nominal trajectory in some near future [5]. A drawback with path following is that it only uses the position to correct the trajectory but it neglects the velocity, which means that the projectile will fly through the nominal trajectory and further corrections are needed. Trajectory integration is one of the impact points prediction, which makes use of numerical calculation of the trajectory to get the impact point. The predictive accuracy depends on the trajectory model, and the higher accuracy of the model, the higher precision of the predictive. The disadvantage of this method is that for long range shoots of the spin-stability the trajectory is complex, which will cause a large amount of calculation [21, 22]. Proportional navigation is a useful guidance method. But to our best knowledge, only projectiles with jet thrusters as actuators have taken this method $[12,13]$, and if the method can be used for other two concepts is needed to be researched. Of course, some new methods are proposed for guidance $[23,24]$. Perturbation based guidance for a generic $2 \mathrm{D}$ trajectory correction fuze is proposed by Robinson, which uses perturbation theory to predict state variables of the projectile [25]. The advantage is that the onboard computer can compute the control signal quickly by the parameters loaded prior to flight, but there is still a drawback that the guidance algorithm only takes drag force in consideration.

In this paper, guidance and control algorithm for a class of spin-stabilized projectile with trajectory correction fuze is proposed. Section 2 presents the trajectory model of this projectile. Section 3 studies the response of trajectory correction. Section 4 introduces a new impact point prediction methods and its application. Section 5 describes the process of guidance and control, and conclusion is provided in Section 6.

\section{Trajectory Model}

Trajectory correction fuze is attached to the projectile by screw thread, and by the bears in the fuze the guided projectile is divided into two parts: the front part called correction part and the aft part called body part. On the correction part, two sets of fixed canards are mounted; one has the same cant angle called steering canards to provide control force, and the other called rotation canards has the

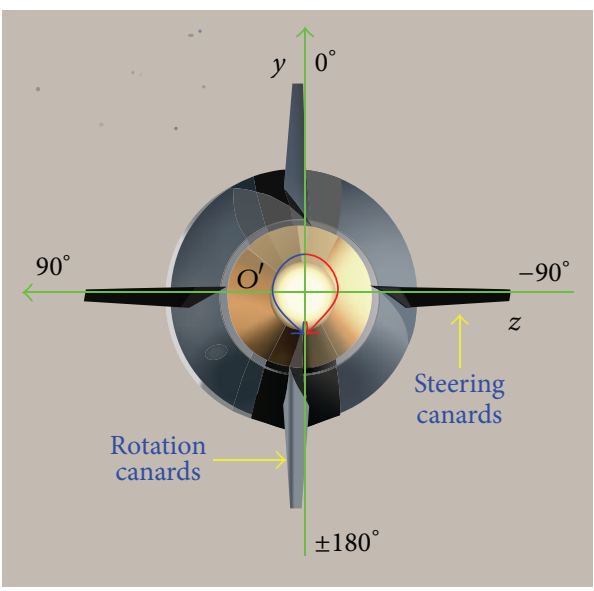

FIGURE 1: Sketch map of correction part.

opposite cant angle to rotate the correction part by the rotational moment, as is shown in Figure 1.

The definition for the roll angle of the correction part is also given in Figure 1. Define the roll angle to be 0 deg, when the steering canards are in horizontal plane and provide upward force, whereas when providing downward force, the roll angle is at the position of $180 \mathrm{deg}$. And the positive direction of the correction part is left, when looking from nose to the base.

2.1. Reference Coordinate System. Two reference coordinate systems are needed in the present analysis: ground launch system and quasi body system. The ground launch frame is an approximation of the earth inertial reference frame. It is a fixed, nonrotating frame, and it neglects the earth's curvature and rotation. Its $x$ and $z$ axes are in the horizontal plane and its $y$-axis is normal to the $x$ - $z$ plane, pointing upward. The original is set at the end of the gun tube, and the $x$ axis points ahead along the launching direction, while the $z$-axis points to the right when looking forward from the gun.

The quasi body reference frame $O x_{4} y_{4} z_{4}$ is fixed to the projectile with its original at the projectile center of mass. Its $x_{4}$-axis aligns with the centerline of the projectile and points forward out of the nose, its positive $z_{4}$-axis to the right, and its $y_{4}$-axis upward. Note that $x_{4}-y_{4}$ plane is fixed in the vertical plane, and it is more convenient to describe the motion of the projectile. The sequence of rotation from the ground launch reference is pitch $\varphi$, yaw $\psi$, as is shown in Figure 2.

2.2. Trajectory Model. During the flight, the two parts of the projectile spin in different directions due to the effort of the aerodynamic forces. In order to describe the motion of the projectile, three translational and four rotational rigid body degrees of freedom are introduced. The translational degrees of freedom are the three components of the mass center position vector. The rotational degrees of freedom are the Euler yaw and pitch angles as well as the correction part roll and body part roll angles. 


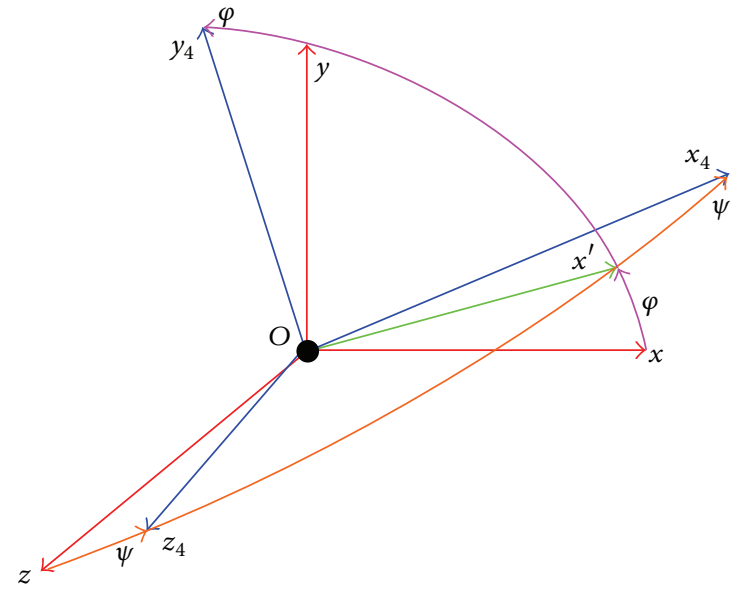

FIGURE 2: Coordinate transformation relation.

Equations (1)-(4) represent the translational and rotational kinematic and dynamic equations of motion for a dual spin projectile, and all these equations are expressed in the quasi body reference frame:

$$
\begin{aligned}
\frac{\mathrm{d} v_{x 4}}{\mathrm{~d} t}+v_{z 4} \omega_{z 4}-v_{y 4} \omega_{y 4} & =\frac{F_{x 4}}{m}, \\
\frac{\mathrm{d} v_{y 4}}{\mathrm{~d} t}+v_{x 4} \omega_{y 4}+v_{z 4} \omega_{z 4} \tan \psi & =\frac{F_{y 4}}{m}, \\
\frac{\mathrm{d} v_{z 4}}{\mathrm{~d} t}-v_{x 4} \omega_{z 4}-v_{y 4} \omega_{y 4} \tan \psi & =\frac{F_{z 4}}{m} .
\end{aligned}
$$

$F_{x 4}, F_{y 4}, F_{z 4}$ are components of the total force expressed in the quasi body reference frame, $v_{x 4}, v_{y 4}, v_{z 4}$ are velocity vector components of the composite center of mass expressed in the quasi body frame, $\omega_{y 4}, \omega_{z 4}$ are components of the angular velocity vector expressed on the $y$ - and $z$-axis of the quasi body reference frame, $m$ is the mass of the projectile, and $\psi$ is the yaw angle:

$$
\left[\begin{array}{c}
\dot{x} \\
\dot{y} \\
\dot{z}
\end{array}\right]=\left[\begin{array}{ccc}
\cos \varphi \cos \psi & \sin \varphi \cos \psi & -\sin \psi \\
-\sin \varphi & \cos \varphi & 0 \\
\cos \varphi \sin \psi & \sin \varphi \sin \psi & \cos \psi
\end{array}\right]\left[\begin{array}{c}
v_{x 4} \\
v_{y 4} \\
v_{z 4}
\end{array}\right] .
$$

$x, y, z$ are position vector components of the composite center of mass expressed in the inertial frame and $\varphi$ is the pitch angle

$$
\begin{aligned}
{\left[\begin{array}{c}
J_{x f} \dot{\omega}_{x f} \\
J_{x} \dot{\omega}_{x 4} \\
J_{y} \dot{\omega}_{y 4} \\
J_{z} \dot{\omega}_{z 4}
\end{array}\right]=} & {\left[\begin{array}{c}
M_{x f} \\
M_{x} \\
M_{y} \\
M_{z}
\end{array}\right]-\left[\begin{array}{c}
0 \\
\left(J_{z}-J_{y}\right) \omega_{y 4} \omega_{z 4} \\
\left(J_{x}-J_{z}\right) \omega_{x 4} \omega_{z 4} \\
\left(J_{y}-J_{x}\right) \omega_{x 4} \omega_{y 4}
\end{array}\right] } \\
& +\left[\begin{array}{c}
0 \\
0 \\
-J_{z} \omega_{z 4} \dot{\gamma}_{a} \\
J_{y} \omega_{y 4} \dot{\gamma}_{a}
\end{array}\right] .
\end{aligned}
$$

$J_{x f}, J_{x}$ are moments of both the front and the aft parts expressed on the $x$-axis of the quasi body reference frame, $J_{y}, J_{z}$ are moments of the projectile expressed on the $y$ and $z$-axis of the quasi body reference frame, $\omega_{x f}, \omega_{x 4}$ are components of the angular velocity vector of both the front and the aft parts expressed on the $x_{4}$-axis of the quasi body reference frame, $\omega_{y 4}, \omega_{z 4}$ are components of the angular velocity vector expressed on the $y$-and $z$-axis of the quasi body reference frame, $M_{x f}, M_{x}$ are components of the total moment of both the front and the aft parts expressed on the $x$-axis of the quasi body reference frame, $M_{y}, M_{z}$ are components of the total moment expressed on the $y$ - and $z$-axis of the quasi body reference frame, and $\gamma_{f}, \gamma_{a}$ are roll angles of the correction and body part:

$$
\left[\begin{array}{c}
\dot{\varphi} \\
\dot{\psi} \\
\dot{\gamma}_{a} \\
\dot{\gamma}_{f}
\end{array}\right]=\left[\begin{array}{cccc}
0 & \frac{\sin \gamma}{\cos \psi} & \frac{\cos \gamma_{\gamma}}{\cos \psi} & 0 \\
0 & \cos \gamma_{a} & -\sin \gamma_{a} & 0 \\
1 & \tan \psi \sin \gamma_{a} & \tan \psi \cos \gamma_{a} & 0 \\
0 & 0 & 0 & 1
\end{array}\right]\left[\begin{array}{c}
\omega_{x 4} \\
\omega_{y 4} \\
\omega_{z 4} \\
\omega_{x f}
\end{array}\right] .
$$

Loads on the composite projectile body are due to weight and aerodynamic forces. All the aerodynamic coefficients are acquired by numerical computing, and the forces as well as moments present as follows:

$$
\begin{aligned}
F_{x 4}= & -\frac{1}{2} \rho v^{2} S C_{a}, \\
F_{y 4}= & \frac{1}{2} \rho v^{2} S C_{n}^{\prime} \alpha-\frac{1}{2} \rho v^{2} S C_{z f}^{\prime \prime} \frac{d \dot{\gamma}_{f}}{v} \beta \\
& -\frac{1}{2} \rho v^{2} S C_{z a}^{\prime \prime} \frac{d \dot{\gamma}_{a}}{v} \beta \\
& +\frac{1}{2} \rho v^{2} S C_{n \delta}\left(\delta_{z} c_{\gamma_{f}}+\alpha\right), \\
F_{z 4}= & -\frac{1}{2} \rho v^{2} S C_{n}^{\prime} \beta-\frac{1}{2} \rho v^{2} S C_{z f}^{\prime \prime} \frac{d \dot{\gamma}_{f}}{v} \alpha \\
& -\frac{1}{2} \rho v^{2} S C_{z a}^{\prime \prime} \frac{d \dot{\gamma}_{a}}{v} \alpha \\
& -\frac{1}{2} \rho v^{2} S C_{n \delta}\left(-\delta_{z} s_{\gamma_{f}}+\beta\right) \\
M_{x}= & -\frac{1}{2} \rho v^{2} S L \frac{d p a}{v} C_{l p a}-M_{a f}, \\
M_{x f}= & \frac{1}{2} \rho v^{2} S L m_{x h}-\frac{1}{2} \rho v^{2} S L \frac{d p}{v} C_{l p f}-M_{f a},
\end{aligned}
$$




$$
\begin{aligned}
M_{y}= & \frac{1}{2} \rho v^{2} S L m_{z}^{\prime} \beta-\frac{1}{2} \rho v^{2} S L C_{n r} \frac{L r}{v} \\
& +\frac{1}{2} \rho v^{2} S L C_{n p a f} \frac{d \dot{\gamma}_{f}}{v} \alpha+\frac{1}{2} \rho v^{2} S L C_{n p a a} \frac{d \dot{\gamma}_{a}}{v} \alpha \\
& +\frac{1}{2} \rho v^{2} S L_{C} C_{n \delta}\left(-\delta_{z} c_{\gamma_{f}}+\beta\right), \\
M_{z}= & \frac{1}{2} \rho v^{2} S L m_{z}^{\prime} \alpha-\frac{1}{2} \rho v^{2} S L C_{m q} \frac{L q}{v} \\
& -\frac{1}{2} \rho v^{2} S L C_{n p a f} \frac{d \dot{\gamma}_{f}}{v} \beta-\frac{1}{2} \rho v^{2} S L C_{n p a a} \frac{d \dot{\gamma}_{a}}{v} \beta \\
& +\frac{1}{2} \rho v^{2} S L_{C} C_{n \delta}\left(\delta_{z} s_{\gamma_{f}}+\alpha\right) .
\end{aligned}
$$

$C_{a}$ is axial force coefficient, $C_{n}^{\prime}$ is normal force derivative coefficient, $C_{z f}^{\prime \prime}, C_{z a}^{\prime \prime}$ are Magnus force coefficient of the correction and body part, $m_{x h}$ is roll moment coefficient of the front part, $m_{z}^{\prime}$ is overturn moment coefficient, $C_{l p f}, C_{l p a}$ are roll damping moment coefficient of the correction and body part, $C_{n r}, C_{m q}$ are yaw and pitch rate damping moment coefficient, $C_{n p a f}, C_{n p a a}$ are Magnus moment aerodynamic coefficient of the correction and body part, $\alpha, \beta$ are attack and sideslip angles, $S, L$ are reference area and length, $d$ is diameter of the projectile, and $v$ is the total velocity. The attack and sideslip angles are computed as follows:

$$
\begin{aligned}
& \alpha=-\tan ^{-1}\left(\frac{v_{y 4}}{v}\right), \\
& \beta=\tan ^{-1}\left(\frac{v_{z 4}}{v}\right) .
\end{aligned}
$$

When the projectile is controlled, the correction part will be set at a given command angle relative to the ground launch frame, and the roll velocity of the correction part will be 0 . So the control equations can be added as follows:

$$
\begin{aligned}
& \gamma_{f}=\gamma_{c}, \\
& \dot{\gamma}_{f}=\omega_{x f}=0,
\end{aligned}
$$

where $\gamma_{f}$ and $\omega_{x f}$ are the roll angle and roll angle velocity components on the $x_{4}$-axis of the quasi body frame and $\gamma_{c}$ is the command angle.

\section{Control Mechanism Strategy}

In this section, control mechanism strategy is researched. Firstly, formula for dynamic equilibrium angle caused by the trajectory correction is deduced based on the equations of the angle motion. Then, swerve response is studied by researching the deviation motion, and the formulas for the magnitude and the phase angle are acquired. Finally, formula for the phase angle of a spin-stability projectile with Mach number as argument is got.
3.1. Dynamic Equilibrium Angle Caused by Correction. When deducing the equation of angle motion, a few assumptions are invoked.

(1) The argument is changed from time $t$ to arc length $s$, and the arc length is defined as follows:

$$
s=\int_{0}^{t} v \mathrm{~d} \tau
$$

(2) The attack and sideslip angles are small, so

$$
\begin{aligned}
& \alpha \approx-\frac{v_{y 4}}{v}, \\
& \beta \approx \frac{v_{z 4}}{v} .
\end{aligned}
$$

(3) Compared to $v_{x 4}, \omega_{x 4}$, and $\omega_{x h}$, the quantities $v_{y 4}, v_{z 4}, \omega_{y 4}$, and $\omega_{z 4}$ are small, so that the total velocity

$$
\dot{v} \approx \dot{v}_{x 4}=-\frac{\rho S C_{a}}{2 m} v^{2}-g \sin \varphi \cos \psi
$$

Products of small quantities and their derivatives are negligible.

(4) The projectile is mass balanced, such that the centers of gravity of both the correction part and the body part lie on the rotational axis of symmetry:

$$
J_{z}=J_{y}
$$

Neglecting the products of small quantities, the derivation of (10) is that

$$
\begin{aligned}
& \dot{\alpha}=-\frac{F_{y 4}}{m v}+\omega_{z 4}-\frac{\dot{v}}{v} \alpha, \\
& \dot{\beta}=\frac{F_{z 4}}{m v}+\omega_{y 4}-\frac{\dot{v}}{v} \beta .
\end{aligned}
$$

Let $\Delta=\alpha-i \beta, \mu=\omega_{y 4}+i \omega_{z 4}, J_{y}=J_{z}=A$; substituting (5) into (13), after a series of deducing, the equation of angle motion is acquired:

$$
\Delta^{\prime \prime}+(H-i P) \Delta^{\prime}-(M+i P T) \Delta=G+D
$$


$G$ and $D$ are the gravity term and control force term, respectively, and the meaning of the symbols is as follows:

$$
\begin{aligned}
& H=k_{z z}+b_{y}-b_{x}-\frac{g}{v^{2}} \sin \varphi \cos \psi+b_{c} \\
& P=\frac{b_{z f} \dot{\gamma}_{f}+b_{z a} \dot{\gamma}_{a}}{v}+\frac{J_{x f} \dot{\gamma}_{f}+J_{x a} \dot{\gamma}_{a}}{A v}
\end{aligned}
$$

M

$$
\begin{gathered}
=k_{z}-k_{z z}\left(b_{y}-b_{x}-\frac{g}{v^{2}} \sin \varphi \cos \psi+b_{c}\right) \\
+\frac{\left(J_{x f} \dot{\gamma}_{f}+J_{x a} \dot{\gamma}_{a}\right)\left(b_{z f} \dot{\gamma}_{f}+b_{z a} \dot{\gamma}_{a}\right)}{A v^{2}}+k_{c}
\end{gathered}
$$

PT

$$
\begin{gathered}
=\frac{J_{x f} \dot{\gamma}_{f}+J_{x a} \dot{\gamma}_{a}}{A v}\left(b_{y}-b_{x}-\frac{g}{v^{2}} \sin \varphi \cos \psi+b_{c}\right) \\
+\frac{k_{z z}\left(b_{z f} \dot{\gamma}_{f}+b_{z a} \dot{\gamma}_{a}\right)}{v}-\frac{\left(k_{y f} \dot{\gamma}_{f}+k_{y a} \dot{\gamma}_{a}\right)}{v},
\end{gathered}
$$

G

$$
\begin{aligned}
= & \frac{g}{v^{2}}(\cos \varphi+i \sin \varphi \sin \psi)\left(k_{z z}-i \frac{J_{x f} \dot{\gamma}_{f}+J_{x a} \dot{\gamma}_{a}}{A v}\right), \\
D & =\left[k_{c}-b_{c}\left(k_{z z}-i \frac{J_{x f} \dot{\gamma}_{f}+J_{x a} \dot{\gamma}_{a}}{A v}\right)\right] \delta_{z} e^{i \gamma_{f}}, \\
k_{y a} & =\frac{\rho S L d C_{n p a a}}{2 A}, \\
k_{c}= & \frac{\rho S L_{c} C_{n \delta}}{2 A}, \\
b_{x}= & \frac{\rho S C_{a}}{2 m}, \\
b_{y}= & \frac{\rho S C_{n}^{\prime}}{2 m}, \\
b_{z f} & =\frac{\rho S d C_{z f}^{\prime \prime}}{2 m}, \\
k_{z z} & =\frac{\rho S C_{z a}^{\prime \prime} C_{m q}}{2 m}, \\
k_{z}= & \frac{\rho S L m_{n \delta}^{\prime}}{2 m}, \\
b_{z} &
\end{aligned}
$$

Coefficients frozen method is taken here, which means that all the aerodynamic coefficients will not change in the near future. So (14) is a linear differential equation, and its analytic solution can be obtained. But here the gravity term will not be considered, because this paper just studies the swerve response of correction control, so the following equation is got:

$$
\Delta^{\prime \prime}+(H-i P) \Delta^{\prime}-(M+i P T) \Delta=D
$$

All the following research is based on the assumption that the projectile is stable. From (16), we know that the angle motion caused by correction control can be divided into fast and slow modes of oscillation, and the oscillation will decay as the projectile flies downrange, because the real parts of the eigenvalues are negative. So the particular solution is only considered, which is called dynamic equilibrium angle caused by correction control.

Define $\Delta_{0 c}$ as a particular solution for (16), and

$$
\Delta_{0 c}=c_{1} e^{l_{1} s}+c_{2} e^{l_{2} s}
$$

$l_{1}$ and $l_{2}$ are roots for homogeneous equation, so

$$
\begin{aligned}
l_{1}+l_{2} & =-(H-i P), \\
l_{1} l_{2} & =-(M+i P T) .
\end{aligned}
$$

Let $c_{1}^{\prime} e^{l_{1} s}+c_{2}^{\prime} e^{l_{2} s}=0$ and differentiate $\Delta_{0 c}$. After deducing, we get

$$
\begin{aligned}
c_{1,2}^{\prime} & = \pm \frac{G}{l_{1}-l_{2}} e^{-l_{1,2} s} \\
& = \pm \frac{1}{l_{1}-l_{2}} \frac{g \cos \varphi}{v^{2}}\left(k_{z z}-i \frac{J_{x f} \dot{\gamma}_{f}+J_{x a} \dot{\gamma}_{a}}{A v}\right) e^{-l_{1,2} s}
\end{aligned}
$$

Let $Q^{*}=k_{z z}-i\left(J_{x f} \dot{\gamma}_{f}+J_{x a} \dot{\gamma}_{a}\right) / A v$. The quantity of $Q^{*}$ is small and can be seen as a constant in a small time period. Integrating the $c_{1,2}^{\prime}$, we have

$$
c_{1,2}= \pm \frac{g Q^{*}}{l_{1}-l_{2}} \frac{1}{-l_{1,2}} \frac{\cos \varphi}{v^{2}} e^{-l_{1,2} s} .
$$

Substituting (17) into (16), it gives

$$
\Delta_{0 c}=-\frac{\left(k_{c}-b_{c} k_{z z}\right)+i b_{c}\left(J_{x a} \dot{\gamma}_{a}\right) /(A v)}{M+i P T} \delta_{z} e^{i \gamma_{f}}
$$

3.2. Swerve Response of Spin-Stability Projectiles. The point mass trajectory model is the first order approximation of the real trajectory, which only takes the gravity as well as the zero yaw axial force in consideration. In the flight, other aerodynamic forces and moments act on the projectile and make the flight contrail diverge from the point 
mass trajectory. The motion which is perpendicular to the point mass trajectory is called the deviation motion [26].

Define the components of the deviation motion as $y_{p}$ and $z_{p}$, so we have

$$
\begin{aligned}
\ddot{y}_{p}+i \ddot{z}_{p}= & \frac{\left(F_{y 4}+i F_{z 4}\right)}{m} \\
= & \left(b_{y}+b_{c}+i \frac{b_{z f} \dot{\gamma}_{f}+b_{z a} \dot{\gamma}_{a}}{v}\right) v^{2} \Delta \\
& +b_{c} v^{2} \delta_{z} e^{i \gamma_{f}}-g \cos \varphi .
\end{aligned}
$$

Taking the arc length $s$ as argument and taking $\dot{\gamma}_{f}=\omega_{x f}=0$, it gives

$$
y_{p}^{\prime \prime}+i z_{p}^{\prime \prime}=\left(b_{y}+b_{c}-i \frac{b_{z a} \dot{\gamma}_{a}}{v}\right) \Delta+b_{c} \delta_{z} e^{i \gamma_{c}}-\frac{g \cos \varphi}{v^{2}}
$$

Neglecting the third term which is small and substituting (21) into (23), it gives

$$
\begin{gathered}
v_{y p}+i v_{z p}=\int\left[-\left(b_{y}+b_{c}-i \frac{b_{z a} \dot{\gamma}_{a}}{v}\right)\right. \\
\cdot \frac{\left(k_{c}-b_{c} k_{z z}\right)+i b_{c}\left(J_{x a} \dot{\gamma}_{a}\right) /(A v)}{M+i P T} \delta_{z} e^{i \gamma_{c}} \\
\left.+b_{c} \delta_{z} e^{i \gamma_{c}}\right] \mathrm{d} s, \\
y_{p}+i z_{p}=\iint\left[-\left(b_{y}+b_{c}-i \frac{b_{z a} \dot{\gamma}_{a}}{v}\right)\right. \\
\cdot \frac{\left(k_{c}-b_{c} k_{z z}\right)+i b_{c}\left(J_{x a} \dot{\gamma}_{a}\right) /(A v)}{M+i P T} \delta_{z} e^{i \gamma_{c}} \\
\left.+b_{c} \delta_{z} e^{i \gamma_{c}}\right] \mathrm{d} s \mathrm{~d} s .
\end{gathered}
$$

Con_real

$$
\begin{aligned}
& =\frac{\left[\left(b_{y}+b_{c}\right)\left(-k_{c}+b_{c} k_{z z}\right)-\left(b_{z a} \dot{\gamma}_{a} / v\right) b_{c}\left(J_{x a} \dot{\gamma}_{a} / A v\right)\right] M+\left[\left(b_{z a} \dot{\gamma}_{a} / v\right)\left(k_{c}-b_{c} k_{z z}\right)-\left(b_{y}+b_{c}\right) b_{c}\left(J_{x a} \dot{\gamma}_{a} / A v\right)\right] P T}{M^{2}+P^{2} T^{2}} \\
& +b_{c}
\end{aligned}
$$

Con_imag

$$
=\frac{\left[\left(b_{y}+b_{c}\right)\left(-k_{c}+b_{c} k_{z z}\right)-\left(b_{z a} \dot{\gamma}_{a} / v\right) b_{c}\left(J_{x a} \dot{\gamma}_{a} / A v\right)\right] P T+\left[\left(b_{z a} \dot{\gamma}_{a} / v\right)\left(k_{c}-b_{c} k_{z z}\right)-\left(b_{y}+b_{c}\right) b_{c}\left(J_{x a} \dot{\gamma}_{a} / A v\right)\right] M}{M^{2}+P^{2} T^{2}}
$$

$v_{y p}, v_{z p}$ and $y_{p}, z_{p}$ are the velocity and position components of the deviation motion, respectively. $\gamma_{c}$ is the command angle. Equation (24) can be changed into

$$
\begin{gathered}
v_{y p}+i v_{z p}=\left[-\left(b_{y}+b_{c}-i \frac{b_{z f} \dot{\gamma}_{f}+b_{z a} \dot{\gamma}_{a}}{v}\right)\right. \\
\left.\cdot \frac{\left(k_{c}-b_{c} k_{z z}\right)+i b_{c}\left(J_{x a} \dot{\gamma}_{a}\right) /(A v)}{M+i P T}+b_{c}\right] \delta_{z} e^{i \gamma_{c}} \int \mathrm{d} s \\
y_{p}+i z_{p}=\left[-\left(b_{y}+b_{c}-i \frac{b_{z f} \dot{\gamma}_{f}+b_{z a} \dot{\gamma}_{a}}{v}\right)\right. \\
\left.\cdot \frac{\left(k_{c}-b_{c} k_{z z}\right)+i b_{c}\left(J_{x a} \dot{\gamma}_{a}\right) /(A v)}{M+i P T}+b_{c}\right] \\
\cdot \delta_{z} e^{i \gamma_{c} \iint \mathrm{d} s \mathrm{~d} s .}
\end{gathered}
$$

The assumption is invoked that velocity and aerodynamic coefficients of the projectile do not change in the small period. $X_{c}$ is the component of the flying path on the $x$-axis of the ground frame, and $\varphi$ is the pitch angle, which is also assuming not change:

$$
\begin{gathered}
\int \mathrm{d} s=s=\frac{X_{c}}{\cos \varphi} \\
\iint \mathrm{d} s \mathrm{~d} s=s^{2}=\left(\frac{X_{c}}{\cos \varphi}\right)^{2} .
\end{gathered}
$$

Let Con $=-\left(b_{y}+b_{c}-i\left(b_{z a} \dot{\gamma}_{a} / v\right)\right)\left(\left(\left(k_{c}-b_{c} k_{z z}\right)+\right.\right.$ $\left.\left.i b_{c}\left(J_{x a} \dot{\gamma}_{a}\right) /(A v)\right) /(M+i P T)\right)+b_{c}$, and the real and imaginary parts are as follows: 


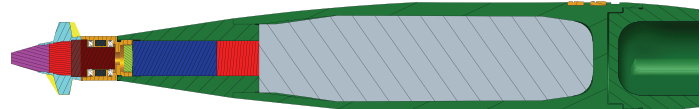

FIGURE 3: A class of spin-stabilized projectile with fixed canards.

Equation (24) can be changed into

$$
\begin{aligned}
v_{y p}+i v_{z p}=\frac{X_{c}}{\cos \varphi} \\
\cdot \delta_{z}\left[\left(\text { Con_real } \cos \gamma_{c}-\text { Con_imag } \sin \gamma_{c}\right)\right. \\
\left.+i\left(\text { Con_real } \sin \gamma_{c}+\text { Con_imag } \cos \gamma_{c}\right)\right], \\
y_{p}+i z_{p}=\left(\frac{X_{c}}{\cos \varphi}\right)^{2} \\
\cdot \delta_{z}\left[\left(\text { Con_real } \cos \gamma_{c}-\text { Con_imag } \sin \gamma_{c}\right)\right. \\
\left.+i\left(\text { Con_real } \sin \gamma_{c}+\text { Con_imag } \cos \gamma_{c}\right)\right] .
\end{aligned}
$$

So we can get magnitudes and phase angle of the velocity and position of the swerve response as follows:

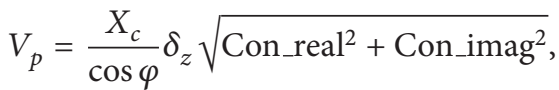

$$
\begin{aligned}
& R_{p}=\left(\frac{X_{c}}{\cos \varphi}\right)^{2} \delta_{z} \sqrt{\text { Con_real }^{2}+\text { Con_imag }^{2}}, \\
& \phi_{p}=\arctan \left(\frac{\text { Con_real } \sin \gamma_{c}+\text { Con_imag } \cos \gamma_{c}}{\text { Con_real } \cos \gamma_{c}-\text { Con_imag } \sin \gamma_{c}}\right) .
\end{aligned}
$$

$V_{p}$ and $R_{p}$ are magnitudes of the velocity and position of the swerve response, whereas $\phi_{p}$ is the phase angle.

3.3. Phase Angle Analysis. The steering canards mounted on the fuze have fixed cant angle, and trajectory correction is realized by adjusting the roll angle of the correction part. So the key point of the control for this class of spin-stabilized projectile is the phase angle between command angle and the direction response.

Take a certain class of spin-stabilized projectile with fixed canards as example, as is shown in Figure 3. Muzzle velocity is $897 \mathrm{~m}-\mathrm{s}^{-1}$, and the mass is $45.5 \mathrm{~kg}$. A simulation experiment is conducted for the phase angle analysis.

The projectile was fired at elevation $45 \mathrm{deg}$, and standard meteorological condition was taken. In simulation correction control began with $10 \mathrm{~s}$, and the command angle was set at 0 deg, $90 \mathrm{deg}, 180 \mathrm{deg}$, and $-90 \mathrm{deg}$, respectively. The results gotten are shown in Figure 4 . The four curves have the same changing trend.

Define $\phi_{d}$ to be the phase shift, which is the angle between the command angle and the direction of the response, and the curves of $\phi_{d}$ are shown in Figure 5. The four curves have small difference, and when the time $t$ is $42.6 \mathrm{~s}$, the biggest magnitude difference is got, which is about 3 deg.

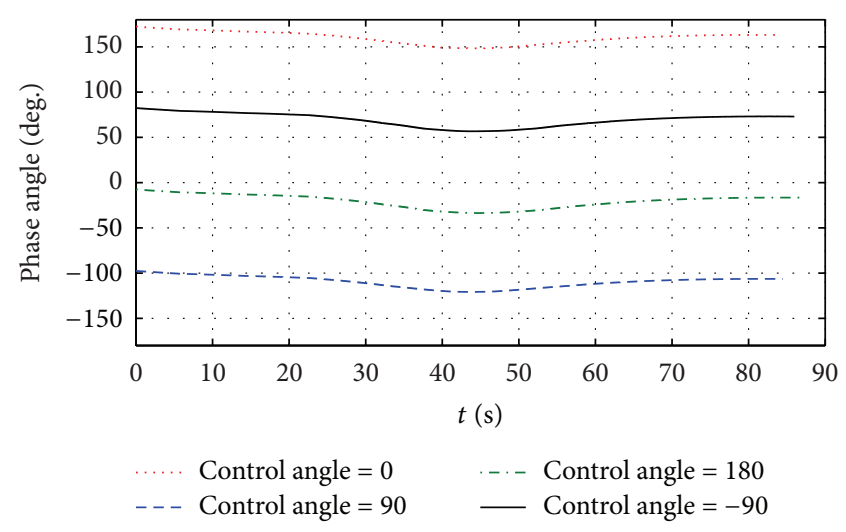

FIGURE 4: Curves of phase angle.

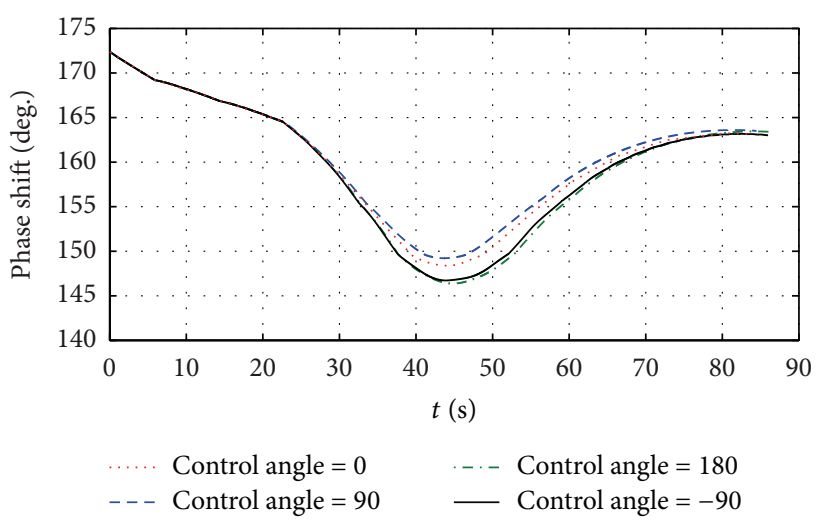

FIgURE 5: Phase shift.

So an assumption is applied, that phase shift for all the command angles is the same one if the state variables of the projectile are given. Since magnitude of $\phi_{p}$ is only relative to velocity and command angle, the phase shift $\phi_{d}$ is a function with Mach number as the argument. Take the method of polynomial fitting (Figure 6), and a formula is got:

$$
\begin{aligned}
\phi_{d}= & -285 \mathrm{Ma}^{4}+1638 \mathrm{Ma}^{3}-3157 \mathrm{Ma}^{2}+3352 \mathrm{Ma} \\
& -1032,
\end{aligned}
$$

where Ma is on behalf of the Mach number.

\section{Impact Point Deviation Prediction Based on Perturbation Theory}

In this section theory of impact point deviation prediction based on perturbation theory is presented firstly. Then the numerical method and application are introduced. At the same time, for projectiles using GPS receiver as the trajectory measurement tool, a second-order fading memory filter is designed. Finally, prediction accuracy is studied by simulation. 


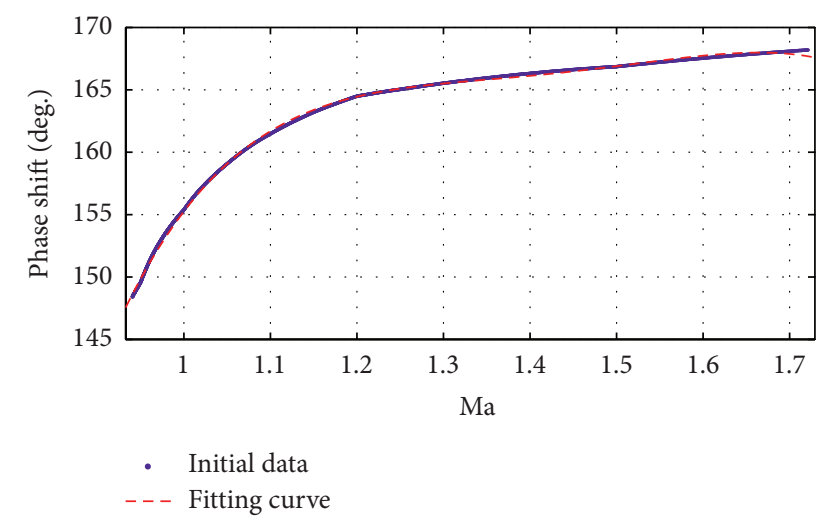

FIgURE 6: Polynomial fitting for phase shift.

4.1. Impact Point Prediction Based on Perturbation Theory. $X_{0}\left(x_{0}, y_{0}, z_{0}\right)$ and $X_{T}\left(x_{T}, y_{T}, z_{T}\right)$ are the positions of the gun and target, respectively. Given the muzzle velocity, the trajectory impacting the target can be acquired definitely. For unguided projectiles the trajectory is the only one, because the range of elevation of the gun is restricted, and the trajectory is called nominal trajectory. Define $X_{T}=\Phi\left(x, y, z, v_{x}, v_{y}, v_{z}\right)$ to be function of nominal trajectory, with $x$ as argument and $v_{x}, v_{y}, v_{z}, y, z$ as state variables.

During flight disturbances always exit, and the projectile deflects from nominal trajectory, which causes the impact point deviation. Define the impact point of flight path influenced by disturbances to be $X_{\operatorname{Im}}\left(x_{\mathrm{Im}}, y_{\mathrm{Im}}, z_{\mathrm{Im}}\right)$. If $X_{\mathrm{Im}}$ is close to target $X_{T}$, perturbation theory can be used to predict the impact point. $X(x, y, z)$ is any point on nominal trajectory, and $X_{\mathrm{Im}}$ can be derived using Taylor expansion:

$$
\begin{gathered}
x_{\operatorname{Im}}=x_{T}+\left[\frac{\partial \Phi_{x}}{\partial v_{x}}, \frac{\partial \Phi_{x}}{\partial v_{y}}, \frac{\partial \Phi_{x}}{\partial v_{z}}, \frac{\partial \Phi_{x}}{\partial y}, \frac{\partial \Phi_{x}}{\partial z}\right] \\
\cdot\left[\Delta v_{x}, \Delta v_{y}, \Delta v_{z}, \Delta y, \Delta z\right]^{T}+R_{x}(X, V), \\
z_{\operatorname{Im}}=z_{T}+\left[\frac{\partial \Phi_{z}}{\partial v_{x}}, \frac{\partial \Phi_{z}}{\partial v_{y}}, \frac{\partial \Phi_{z}}{\partial v_{z}}, \frac{\partial \Phi_{z}}{\partial y}, \frac{\partial \Phi_{z}}{\partial z}\right] \\
\cdot\left[\Delta v_{x}, \Delta v_{y}, \Delta v_{z}, \Delta y, \Delta z\right]^{T}+R_{z}(X, V) .
\end{gathered}
$$

$\Delta v_{x}, \Delta v_{y}, \Delta v_{z}, \Delta y, \Delta z$ are disturbance magnitudes of state variables when range of the projectile is $x$, whereas $R_{x}(X, V)$ and $R_{z}(X, V)$ are Lagrange remainders. If Taylor expansion meets the accuracy requirement, that is to say, the absolute values of $R_{x}(X, V)$ and $R_{z}(X, V)$ are smaller than the given value, (30) can be used to predict the impact point deviation, so we get

$$
\begin{aligned}
D L & =x_{\mathrm{Im}}-x_{T}=\left[\frac{\partial \Phi_{x}}{\partial v_{x}}, \frac{\partial \Phi_{x}}{\partial v_{y}}, \frac{\partial \Phi_{x}}{\partial v_{z}}, \frac{\partial \Phi_{x}}{\partial y}, \frac{\partial \Phi_{x}}{\partial z}\right] \\
\cdot & {\left[\Delta v_{x}, \Delta v_{y}, \Delta v_{z}, \Delta y, \Delta z\right]^{T}, } \\
D H & =z_{\operatorname{Im}}-z_{T}=\left[\frac{\partial \Phi_{z}}{\partial v_{x}}, \frac{\partial \Phi_{z}}{\partial v_{y}}, \frac{\partial \Phi_{z}}{\partial v_{z}}, \frac{\partial \Phi_{z}}{\partial y}, \frac{\partial \Phi_{z}}{\partial z}\right] \\
\cdot & {\left[\Delta v_{x}, \Delta v_{y}, \Delta v_{z}, \Delta y, \Delta z\right]^{T} . }
\end{aligned}
$$

$D L$ and $D H$ are the downrange and lateral deviation of the impact point.

When using this method, note the following.

(1) The impact point $X_{\text {Im }}$ should be close to the target $X_{T}$, and the disturbance magnitudes should not be big ones.

(2) Predictive accuracy should meet requirements.

4.2. Numerical Method and Application. $X_{T}=\Phi(x, y, z$, $\left.v_{x}, v_{y}, v_{z}\right)$ is an implicit function. Taking analytic method to study the predictive accuracy is very hard, while numerical method is a useful way. Numerical method is taken in this paper, and application of partial derivative, nominal trajectory is researched.

In order to predict the deviation in real time, the guidance system needs to acquire the information of velocity and position; at the same time it should get the partial derivatives and disturbance magnitudes. In application, partial derivatives and nominal trajectory are loaded on the onboard computer prior to flight. The information of velocity and position can be acquired by global position system (GPS) receiver, or inertial measurement unit (IMU). The projectile mentioned above takes GPS receiver.

The unguided trajectory whose elevation is $45 \mathrm{deg}$ is used as nominal trajectory, and the trajectory whose elevation is in the neighborhood of $45 \mathrm{deg}$ is used as the disturbance trajectory. The advantage is that the impact point deviation is the same value along the trajectory.

4.2.1. Numerical Computing of Partial Derivatives. Finite difference method is invoked here. Take $\partial \Phi_{x} / \partial v_{x}$ and $\partial \Phi_{z} / \partial v_{x}$ as example. Add disturbance $\Delta v_{x}$ on the state variable $v_{x}$ of any point on the nominal trajectory, and the impact point $X_{\delta}\left(x_{\delta}, y_{\delta}, z_{\delta}\right)$ is got. It gives

$$
\begin{aligned}
& \frac{\partial \Phi_{x}}{\partial v_{x}}=\frac{x_{\delta}-x_{T}}{\Delta v_{x}}, \\
& \frac{\partial \Phi_{z}}{\partial v_{x}}=\frac{z_{\delta}-z_{T}}{\Delta v_{x}} .
\end{aligned}
$$

The second and third order partial derivatives are also computed like this.

Here velocity step and position step are defined, which are velocity and position disturbance. In this part, how to 
TABle 1: Partial derivatives.

\begin{tabular}{lcccccccccc}
\hline Range & $\partial \Phi_{x} / \partial v_{x}$ & $\partial \Phi_{x} / \partial v_{y}$ & $\partial \Phi_{x} / \partial v_{z}$ & $\partial \Phi_{x} / \partial y$ & $\partial \Phi_{x} / \partial z$ & $\partial \Phi_{z} / \partial v_{x}$ & $\partial \Phi_{z} / \partial v_{y}$ & $\partial \Phi_{z} / \partial v_{z}$ & $\partial \Phi_{z} / \partial y$ & $\partial \Phi_{z} / \partial z$ \\
\hline 5146.29 & 26.41 & 26.48 & -4.4 & 1.18 & -0.0013 & -0.31 & 1.25 & 47.98 & 0.069 & 0.99658 \\
6707.75 & 27.53 & 25.95 & -4.45 & 1.04 & -0.0017 & -0.57 & 1.26 & 48.6 & 0.0689 & 0.99688 \\
8144.93 & 27.8 & 25.87 & -4.06 & 0.86 & -0.0021 & -0.84 & 1.26 & 48.38 & 0.062 & 0.99718 \\
9487 & 27.7 & 25.74 & -3.59 & 0.71 & -0.0024 & -1.05 & 1.22 & 47.5 & 0.055 & 0.99747 \\
10753.4 & 28.7 & 26.12 & -3.33 & 0.66 & -0.0028 & -1.09 & 1.13 & 46.2 & 0.055 & 0.99775 \\
11962.3 & 30.35 & 26.06 & -2.99 & 0.62 & -0.003 & -0.91 & 1.01 & 44.2 & 0.056 & 0.99801 \\
13128.3 & 31.0 & 24.94 & -2.96 & 0.604 & -0.0035 & -0.71 & 0.86 & 41.7 & 0.0564 & 0.99825 \\
14260.2 & 30.7 & 23.19 & -2.19 & 0.58 & -0.0039 & -0.46 & 0.75 & 39.2 & 0.057 & 0.99848 \\
15363.2 & 29.74 & 21.79 & -1.6 & 0.57 & -0.0042 & -0.15 & 0.73 & 36.4 & 0.0565 & 0.99870 \\
16440.2 & 28.25 & 20.2 & -1.4 & 0.55 & -0.0045 & -0.62 & 0.81 & 33.4 & 0.053 & 0.99892 \\
17492.6 & 26.19 & 18.31 & -0.87 & 0.54 & -0.0048 & -1.03 & 0.88 & 30.5 & 0.0503 & 0.99913 \\
18519.1 & 23.73 & 16.57 & -0.29 & 0.53 & -0.005 & -1.01 & 0.93 & 27.5 & 0.0495 & 0.999930 \\
19518.4 & 21.6 & 14.4 & -0.33 & 0.52 & -0.005 & -0.82 & 0.84 & 24.5 & 0.0495 & 0.99944 \\
20487.7 & 19.5 & 12.42 & -0.16 & 0.51 & -0.0058 & -0.66 & 0.76 & 21.5 & 0.049 & 0.999574 \\
21422.9 & 17.04 & 10.39 & -0.07 & 0.5 & -0.006 & -0.54 & 0.66 & 18.6 & 0.0486 & 0.99968 \\
22319.2 & 14.62 & 8.83 & -0.15 & 0.49 & -0.006 & -0.42 & 0.59 & 15.6 & 0.0479 & 0.999768 \\
23171.8 & 12.0 & 6.82 & 0.028 & 0.48 & -0.006 & -0.33 & 0.45 & 12.7 & 0.0471 & 0.999838 \\
23975.6 & 9.27 & 4.92 & -0.05 & 0.47 & -0.007 & -0.24 & 0.32 & 9.64 & 0.0463 & 0.999891 \\
24726.5 & 6.32 & 3.42 & 0.17 & 0.46 & -0.007 & -0.16 & 0.23 & 6.51 & 0.0455 & 0.999926 \\
25421.5 & 3.03 & 1.43 & -0.004 & 0.46 & -0.007 & -0.08 & 0.086 & 3.13 & 0.0449 & 0.999943 \\
\hline & & & & & & & & &
\end{tabular}

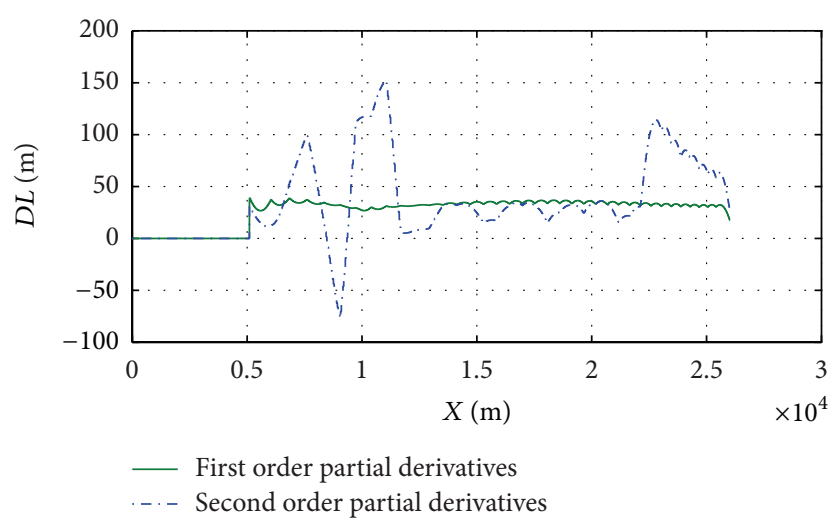

Figure 7: Predictive results with different order partial derivatives.

select the velocity step, position step, and order of the Taylor expansion is presented. Select the trajectory whose elevation is $45.5 \mathrm{deg}$ as disturbance trajectory.

The predictive results of downrange deviation using first and second-order partial derivatives are shown in Figure 7, when velocity step and position step are $2 \mathrm{~m}-\mathrm{s}^{-1}$ and $5 \mathrm{~m}$, and rows of partial derivatives and nominal trajectory are 20 and 75 , respectively. Compared with result of first order, result of second-order has big amplitude, and the convergence is slow. So first order partial derivatives is preferred.

Figure 8 shows the predictive results with different velocity steps and position steps, when rows of partial derivatives and nominal trajectory are 20 and 75, respectively. When position step is $5 \mathrm{~m}$, results are almost the same. But when

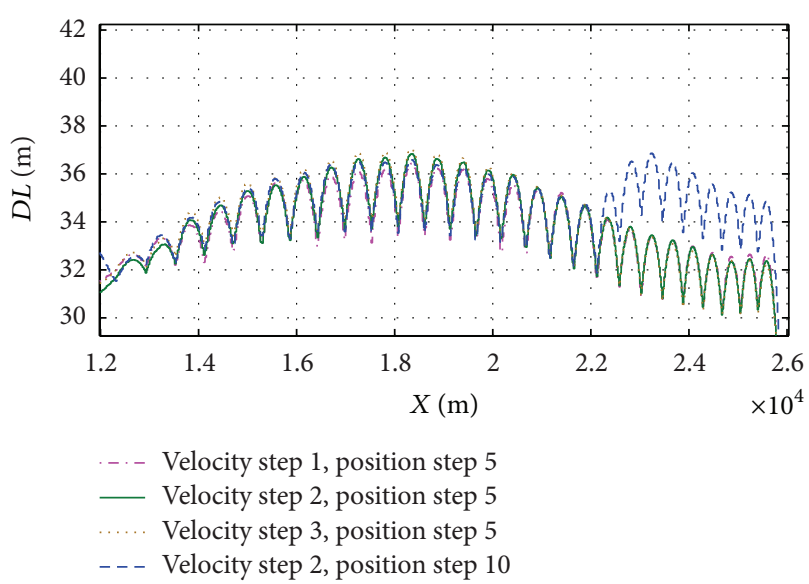

FIGURE 8: Predictive results with different velocity and position step.

velocity step is given as $2 \mathrm{~m}-\mathrm{s}^{-1}$, results have big difference after range reaches $22 \mathrm{~km}$. That is because the impact point is far away from the target, since magnitude of position step is too big. Here we select that velocity step and position step are $2 \mathrm{~m}-\mathrm{s}^{-1}$ and $5 \mathrm{~m}$.

Table 1 shows twenty rows of partial derivatives of nominal trajectory, and values of each line are almost liner. Simulation results show that selecting 20 rows is suitable, since adding rows will not improve the accuracy of prediction.

4.2.2. Numerical Computing of nominal Trajectory. Nominal trajectory loaded on the onboard computer is discrete values 


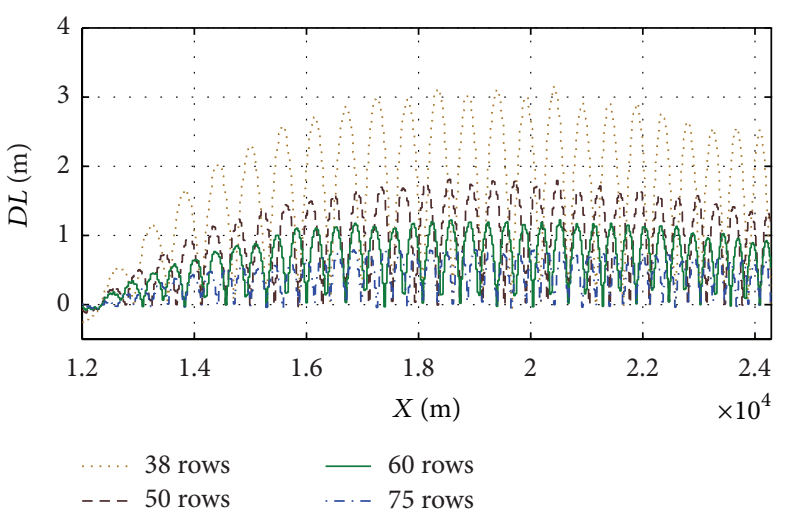

(a) Downrange predictive results

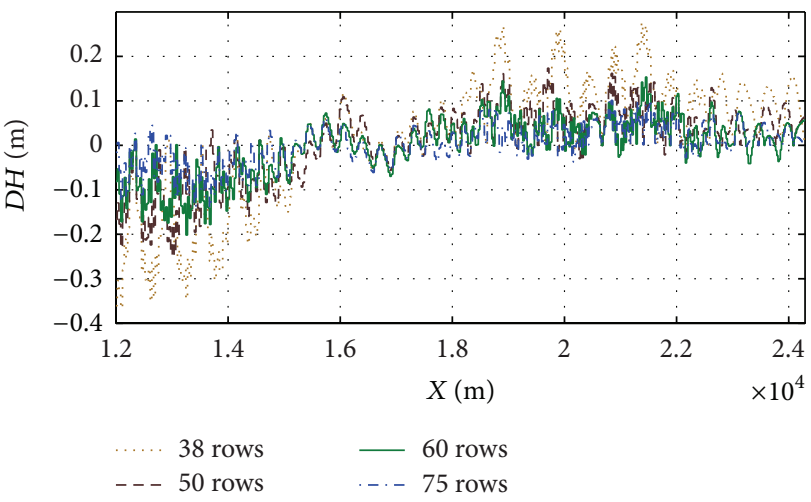

(b) Cross range predictive results

FIGURE 9: Predictive results with different rows of nominal trajectory.

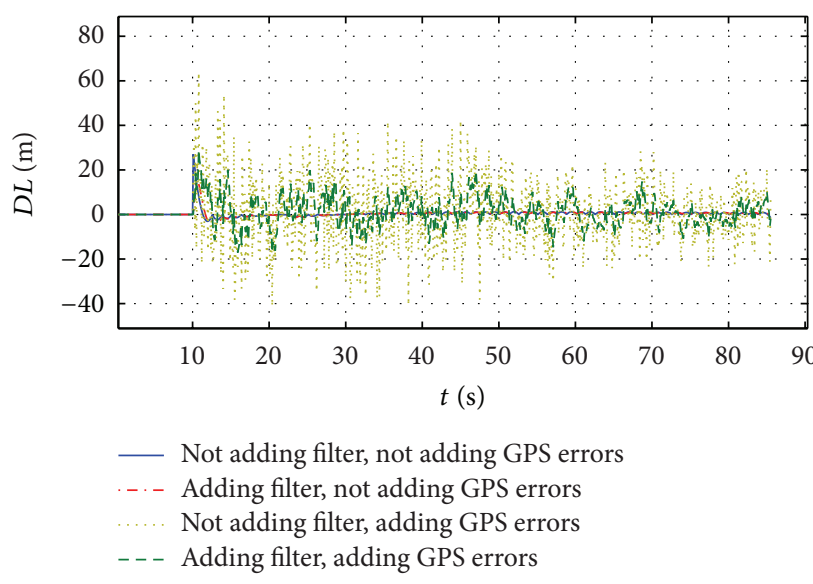

(a) Downrange filter results

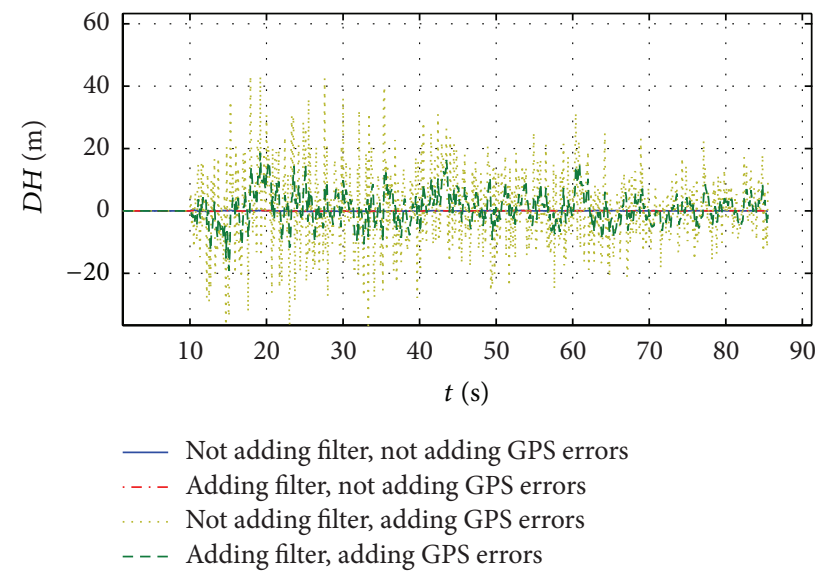

(b) Cross range filter results

FIGURE 10: Results of second-order fading memory filter.

with range $x$ as argument. In application standard value of state variables is acquired by interpolation to the nominal trajectory, and the error between standard value and real value will cause predictive error. Adding rows of nominal trajectory to reduce the range interval is an effective way to improve the predictive accuracy.

Figure 9 shows the results of prediction with different rows of nominal trajectory, when using nominal trajectory as disturbance trajectory. The downrange predictive results change as half sinusoid, and the period is just the sampling period, which means that at the sampling point the predictive error is 0 . The bigger the sampling period is, the bigger the predictive error is. When the rows reach 75 , the downrange error is less than $0.8 \mathrm{~m}$, and the lateral error is less than $0.1 \mathrm{~m}$, whereas, it just occupies no more than $5 \mathrm{k}$ of RAM.

4.3. Fading Memory Filter. GPS receiver has measurement error and will cause predictive error. So a filter is necessary to work in the loop to reduce error. Here second-order fading memory filter is taken, and the algorithm is as follows:

$$
\begin{aligned}
\Delta \widehat{L}_{n}= & \Delta \widehat{L}_{n-1}+\Delta \hat{\dot{L}}_{n-1} T_{s} \\
& +G_{\Delta L}\left[\Delta L_{n}^{*}-\left(\Delta \widehat{L}_{n-1}+\Delta \hat{\dot{L}}_{n-1} T_{s}\right)\right],
\end{aligned}
$$

$$
\begin{aligned}
\Delta \hat{\dot{L}}_{n} & =\Delta \hat{\dot{L}}_{n-1}+\frac{H_{\Delta L}}{T_{s}}\left[\Delta L_{n}^{*}-\left(\Delta \widehat{L}_{n-1}+\Delta \hat{\dot{L}}_{n-1} T_{s}\right)\right], \\
H_{\Delta L} & =\left(1-\beta_{\Delta L}\right)^{2} \\
G_{\Delta L} & =1-\beta_{\Delta L}{ }^{2} .
\end{aligned}
$$

$D \widehat{L}_{n}$ and $D \widehat{L}_{n-1}$ are estimated values of the variable at the current and last instant, $\Delta L_{n}^{*}$ is the measurement value, $D \hat{\dot{L}}_{n}$ and $D \hat{\dot{L}}_{n-1}$ are estimated values of first- and second-order derivatives, $T_{s}$ is sampling instant, $\beta_{\Delta L}$ is gain factor, and $H_{\Delta L}$ and $G_{\Delta L}$ are gain coefficients.

The filter results of the predictive deviation are shown in Figure 10, and the results meet the requirements.

4.4. Prediction Accuracy. As is mentioned above, the unguided trajectory whose elevation is $45 \mathrm{deg}$ is used as nominal trajectory, and the trajectory whose elevation is in the neighborhood of $45 \mathrm{deg}$ is used as the disturbance trajectory. Here trajectory with elevation being $45.5 \mathrm{deg}$ is the disturbance trajectory, and its impact point deviation is a constant, as is shown in Figure 11. Downrange predictive result reflects the real deviation before adding GPS errors, 


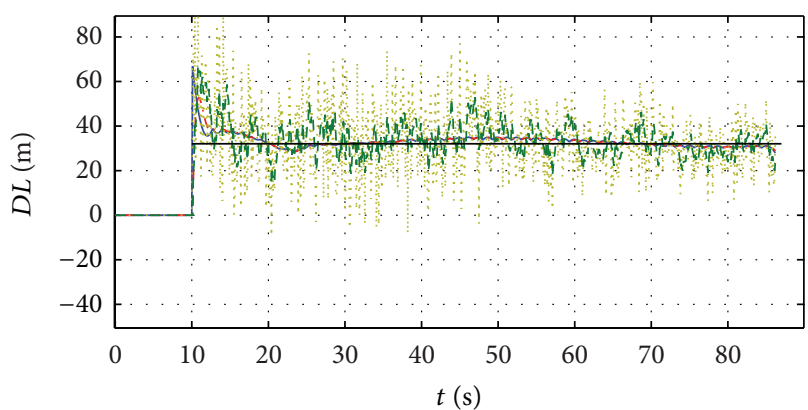

_ Not adding filter, not adding GPS errors
$\ldots$ - Adding filter, not adding GPS errors
$\ldots$. Not adding filter, adding GPS errors
$\ldots-$ Adding filter, adding GPS errors
$\ldots$ Real deviation

(a) Downrange filter results

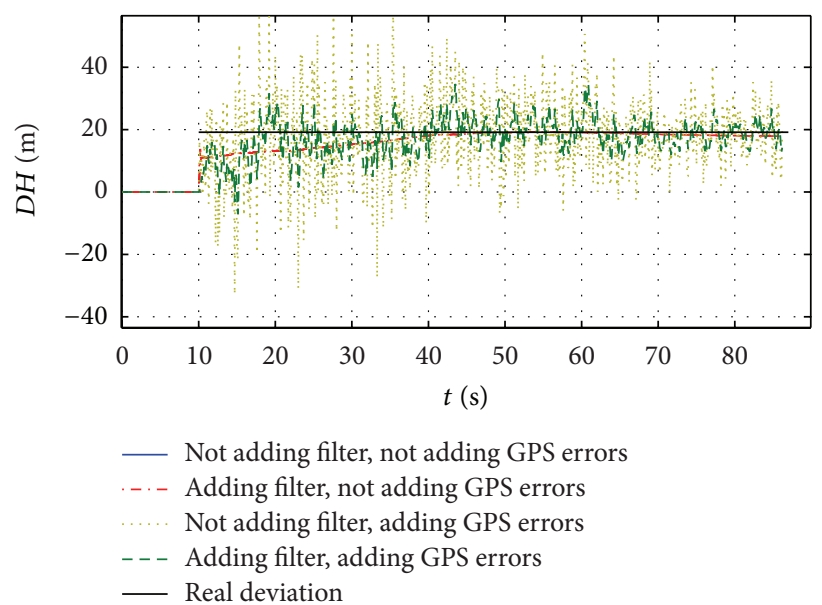

(b) Cross range filter results

FIGURE 11: Results of second-order fading memory filter when fire elevation is $45.5 \mathrm{deg}$.

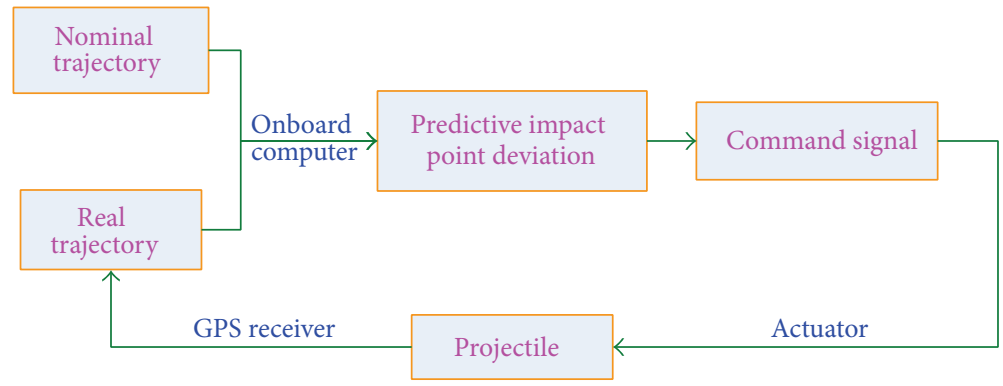

FIgURE 12: Process of guidance and control.

and the predictive error is not more than $2 \mathrm{~m}$. After adding GPS errors, the downrange predictive results also can meet the accuracy requirement. For lateral prediction, till $40 \mathrm{~s}$ the convergence of prediction is got, and the results are aligned with the lateral deviation. Figure 11 presents that the lateral prediction results can describe the deviation; just the errors are a little big, but still not more than $15 \mathrm{~m}$.

Impact point deviation prediction based on perturbation theory for spin-stabilized projectiles has high prediction accuracy and has small calculation amounts. It is suitable for application on the trajectory correction fuze.

\section{Process of Guidance and Control}

For the spin-stabilized projectile mentioned above, the onboard GPS receiver gets the velocity and position information of the real trajectory every $0.1 \mathrm{~s}$ and then onboard computer samples the information, predicts impact point deviation, generates command signal, and controls the actuator to make the correction part steady at a setting angle to correct the real trajectory, which is also a disturbance trajectory, as is shown in Figure 12. The key points are the command signal generation and strategy of guidance and control.

5.1. Command Signal Generation. The objective of correction control is changing the state variables to make the predictive derivation 0 to realize impacting the target precisely, by adjusting the roll angle of steering canards mounted on the correction part. As correction control mechanism is studied in Section 3, the pivotal problem to be researched is the relationship between changes of state variables and impact point derivation.

As is shown in Table 1, factors influencing predictive derivation are velocity and position deviations between the real trajectory and nominal trajectory, which are described in the gound launch frame. The dominating factors of downrange derivation are velocity components deviations on the $x$-axis and $y$-axis, and position components deviation on the $y$-axis, whereas the lateral dominating factors are velocity components deviations on the $x$-axis and $z$-axis, and position components deviation on the $z$-axis. But the changes caused by correction control, called correction values, are presented 
in the quasi body frame, and they are velocity and position components on the $y_{4}$-axis and $z_{4}$-axis, which are defined as $\Delta v_{y 4}, \Delta v_{z 4}, \Delta y_{4}$, and $\Delta z_{4}$. They need to be transformed and presented in the ground launch frame:

$$
\begin{aligned}
& {\left[\begin{array}{l}
\Delta v_{x} \\
\Delta v_{y} \\
\Delta v_{z}
\end{array}\right]} \\
& \quad=\left[\begin{array}{ccc}
\cos \varphi \cos \psi & \sin \varphi \cos \psi & -\sin \psi \\
-\sin \varphi & \cos \varphi & 0 \\
\cos \varphi \sin \psi & \sin \varphi \sin \psi & \cos \psi
\end{array}\right]\left[\begin{array}{c}
0 \\
\Delta v_{y 4} \\
\Delta v_{z 4}
\end{array}\right] \\
& \approx\left[\begin{array}{ccc}
\cos \varphi & \sin \varphi & 0 \\
-\sin \varphi & \cos \varphi & 0 \\
0 & 0 & 1
\end{array}\right]\left[\begin{array}{c}
0 \\
\Delta v_{y 4} \\
\Delta v_{z 4}
\end{array}\right],
\end{aligned}
$$

$$
\begin{aligned}
& {\left[\begin{array}{c}
\Delta x \\
\Delta y \\
\Delta z
\end{array}\right]=\left[\begin{array}{ccc}
\cos \varphi \cos \psi & \sin \varphi \cos \psi & -\sin \psi \\
-\sin \varphi & \cos \varphi & 0 \\
\cos \varphi \sin \psi & \sin \varphi \sin \psi & \cos \psi
\end{array}\right]\left[\begin{array}{c}
0 \\
\Delta y_{4} \\
\Delta z_{4}
\end{array}\right]} \\
& \approx\left[\begin{array}{ccc}
\cos \varphi & \sin \varphi & 0 \\
-\sin \varphi & \cos \varphi & 0 \\
0 & 0 & 1
\end{array}\right]\left[\begin{array}{c}
0 \\
\Delta y_{4} \\
\Delta z_{4}
\end{array}\right] .
\end{aligned}
$$

$\Delta v_{x}, \Delta v_{y}, \Delta v_{z}, \Delta x, \Delta y$, and $\Delta z$ are correction values presented in ground launch frame. $\Delta x$ will be neglected, since it is small. Correction control is to make the real trajectory approach nominal trajectory to reduce the predictive derivation by correction values.

$D L$ and $D H$ are the predictive impact point deviations of downrange and lateral direction. As is shown in Figure 13, the direction of $x_{D}$-axis and $z_{D}$-axis is downrang and lateral direction, respectively. Vector $T X_{\mathrm{im}}$ is the predictive derivation, and angle between the vector and $z_{D}$-axis is

$$
\phi_{D}=\arctan \left(\frac{D L}{D H}\right) .
$$

The direction of swerve response for correction control is the opposite direction of $\phi_{D}$; that is

$$
\phi_{R}=-\phi_{D}=-\arctan \left(\frac{D L}{D H}\right) \text {. }
$$

Since phase lag $\phi_{d}$ exits, the command signal can be computed as follows:

$$
\begin{aligned}
\phi_{C} & =-\arctan \left(\frac{D L}{D H}\right)-\phi_{d} \\
& =\arctan \left(\frac{D L}{D H}\right)+\pi-\phi_{d} .
\end{aligned}
$$

5.2. Control Strategy. As expected, Figure 14 indicates that control authority decreases with the control instant delay, from when the correction part is set at the given roll angle

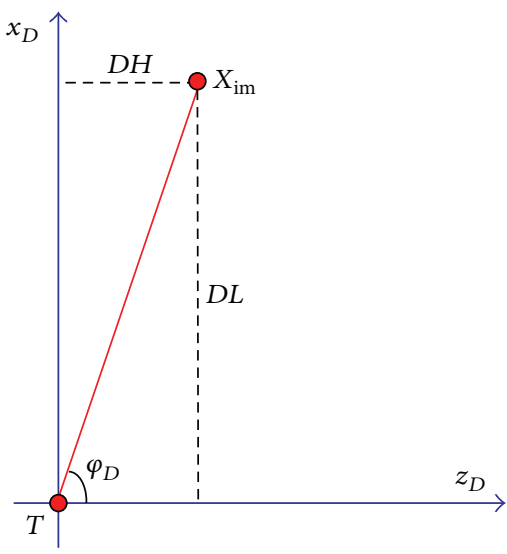

FIGURE 13: Sketch map of predictive derivation.

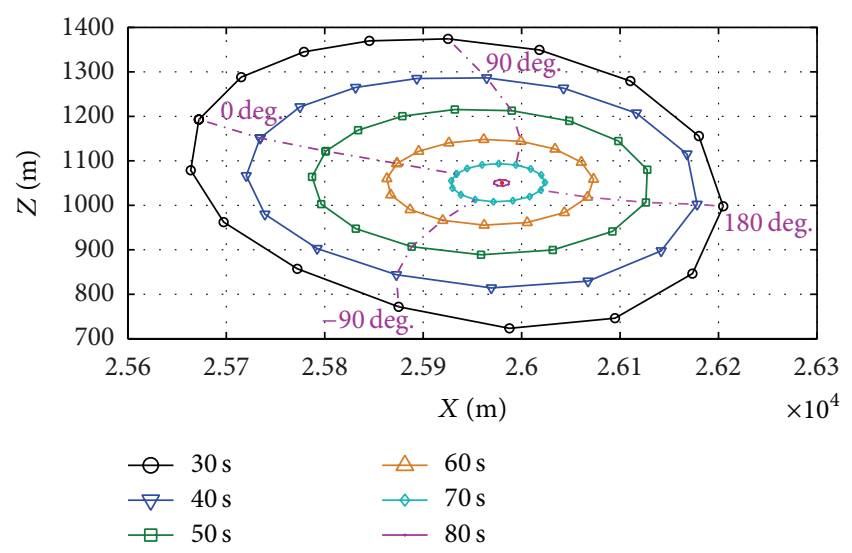

FIGURE 14: Correction authority.

till the projectile impacts the ground. If correction control begins with $30 \mathrm{~s}$, the largest downrange correction authority from far to target is about $260 \mathrm{~m}$, and from near to target is about $300 \mathrm{~m}$, whereas the lateral correction authority is about $260 \mathrm{~m}$.

As is mentioned in Section 5, the predictive derivations have high accuracy after $40 \mathrm{~s}$, and the lateral derivation has predictive errors before $40 \mathrm{~s}$, but it can still reflect the sign of the lateral derivation, which means that from the predictive derivation we can know the predictive impact point is on left or right of the target. Here the control strategy is divided into two segments: in ascending segment only lateral correction is invoked, while comprehensive correction is conducted in descending segment. The command signals in the two segments are shown as follows:

In ascending segment

$$
\begin{aligned}
& \phi_{C}=90-\phi_{z}, \quad \text { if } D H<-S_{z}, \\
& \phi_{C}=-90-\phi_{z}, \quad \text { if } D H>S_{z} .
\end{aligned}
$$

In descending segment

$$
\phi_{C}=\arctan \left(\frac{D L}{D H}\right)+\pi-\phi_{d} .
$$




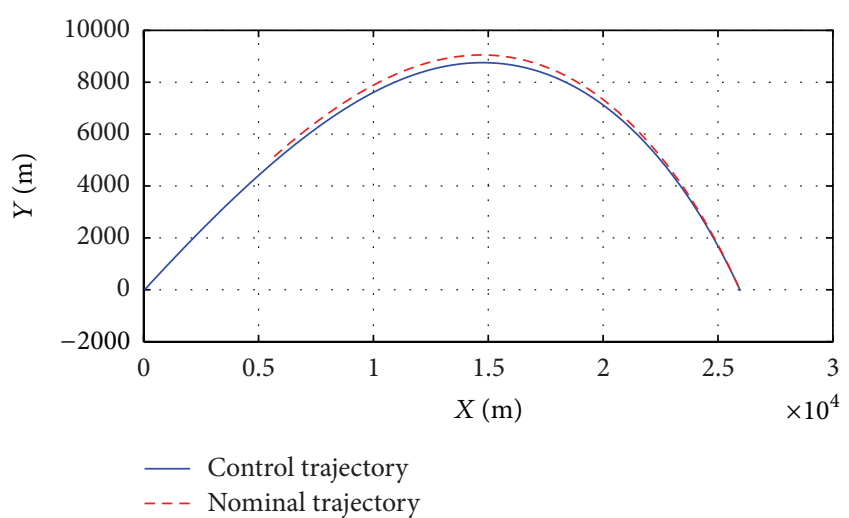

FIGURE 15: Range versus altitude.

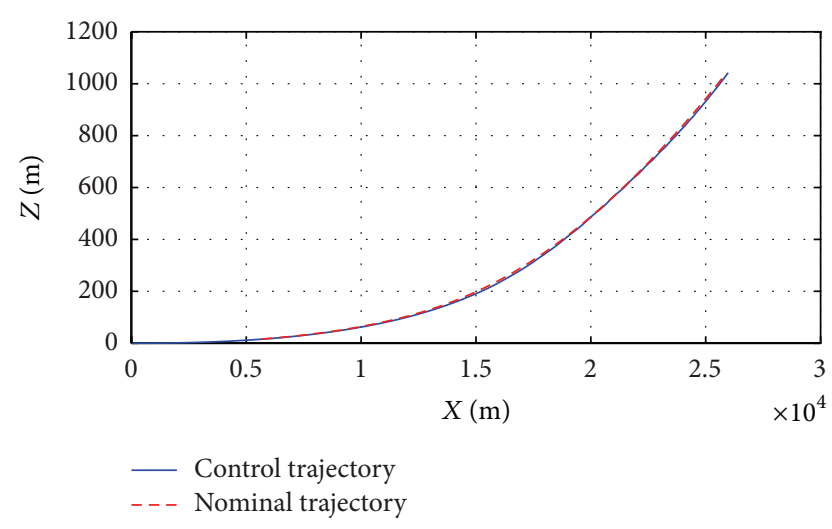

FIGURE 16: Range versus cross range.

\subsection{Simulation Results}

5.3.1. Simulation of the Control Trajectory. In order to validate the effectiveness of the control strategy simulation of control trajectory was conducted with the elevation being $44 \mathrm{deg}$. From Figures 15 and 16, the control trajectory approaches nominal trajectory constantly and impacts the target. Figure 16 indicates that in the lateral plane the control trajectory traverses the nominal trajectory twice. Figures 15 and 16 validate the effectiveness of the control strategy.

Figure 17 presents the result of predictive impact point deviation, and it shows that the two curves are close in upon the target in general trend. There is no gyroscope installed on the guidance and control system, so there is no angular feedback. The control force makes the sideslip angle dither, as is shown in Figure 18, and the control trajectory shows a small wobble, which is reflected in Figure 17. The magnitude of sideslip angle is about $10 \mathrm{deg}$, but after that it declines, which indicates in flight the projectile is kept in stable condition.

When an unguided projectile mentioned above is fired, range and cross range are all smaller than the two of nominal trajectory, and the downrange deviation takes the dominating part. So the phase angle of swerve response needs to be set in the neighborhood of $0 \mathrm{deg}$ to correct the trajectory. Since phase lag exits, command angle should be set at the position

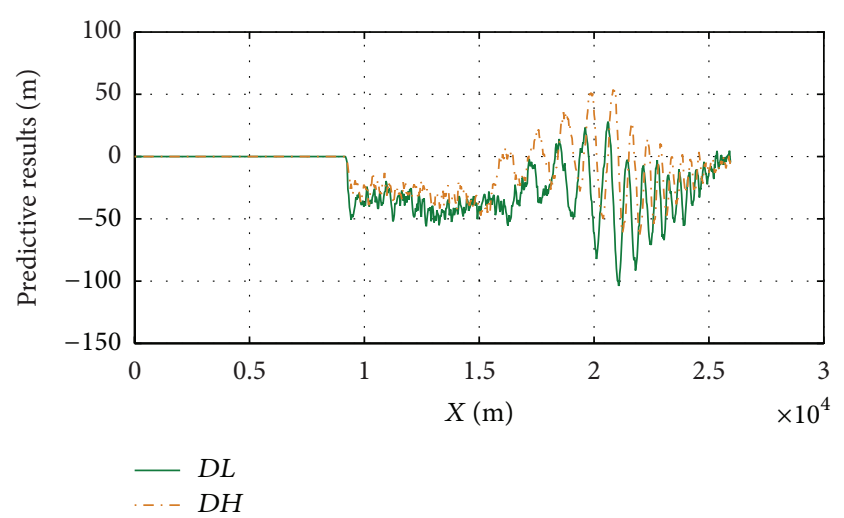

FIgURe 17: Predictive results.

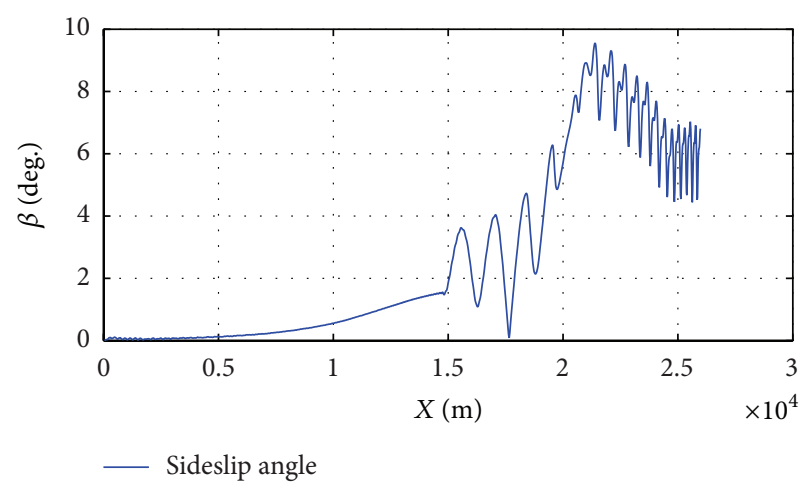

FIGURE 18: Sideslip angle.

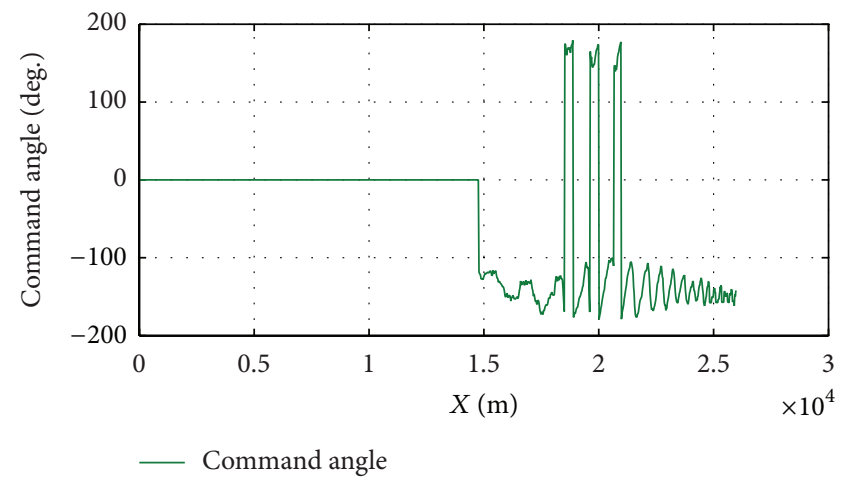

Figure 19: Curve of command angle.

ahead the phase angle of swerve response, as is shown in Figure 19.

5.3.2. Results of Monte Carlo Targeting. Adding all kinds of disturbances, as is shown in Table 2, such as winds, muzzle velocity perturbation, and errors of launching elevation angle, miss distances without guidance and control are shown in Figure 20, which indicates that downrange miss distance is bigger than the lateral one. When guidance and control are taken, the miss distance is much smaller, and CEP is not more than $30 \mathrm{~m}$. Simulation results show that control strategy based on impact point derivation prediction proposed in this 
TABLE 2: Disturbance parameters.

\begin{tabular}{lcc}
\hline Parameter & Unit & Deviation value \\
\hline Mass & $\mathrm{kg}$ & \pm 0.5 \\
Length & $\mathrm{mm}$ & \pm 5 \\
Moment of inertia & $\%$ & \pm 4 \\
Muzzle velocity & $\mathrm{m} / \mathrm{s}$ & \pm 5 \\
Launching elevation angle & $\mathrm{deg}$ & \pm 0.06 \\
Launching azimuth angle & $\mathrm{deg}$ & \pm 0.02 \\
Wind velocity & $\mathrm{m} / \mathrm{s}$ & \pm 2 \\
Wind direction & $\mathrm{deg}$ & \pm 15 \\
\hline
\end{tabular}

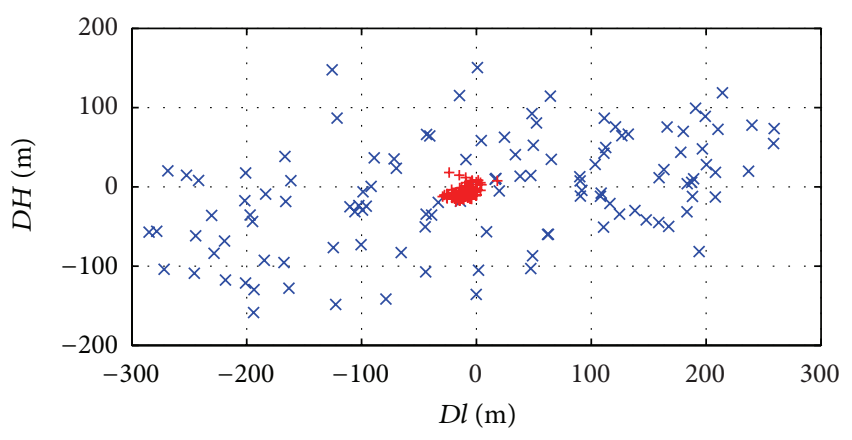

Figure 20: Results of Monte Carlo targeting.

paper is suitable for trajectory correction of this class of spinstabilized projectile.

\section{Conclusions}

Guidance and control strategy for a class of spin-stabilized projectile with $2 \mathrm{D}$ trajectory correction fuze is presented in this paper. Based on correction control mechanism study, control strategy using impact point derivation prediction based on perturbation theory is proposed. Simulation results show that the strategy designed is suitable for this class of projectiles, and the miss distance is reduced effectively.

\section{Conflict of Interests}

The authors declare that there is no conflict of interests regarding the publication of this paper.

\section{References}

[1] J. Clancy, T. Bybee, and W. Friedrih, "Fixed canard 2-D guidance of artillery projectile,” US: 6981672B2 [P], 2006.

[2] M. Costello, "Modeling and simulation of a differential roll projectile," in Proceedings of the AIAA Modeling and Simulation Technologies Conference, pp. 490-499, 1998.

[3] P. Wernert and S. Theodoulis, "Modeling and stability analysis for a class of $155 \mathrm{~mm}$ spin-stabilized projectiles with course correction fuse (CCF)," in Proceedings of the AIAA Atmospheric Flight Mechanics Conference and Exhibit, pp. 1-13, 2011.
[4] S. Theodoulis, V. Gassmann, P. Wernert, L. Dritsas, I. Kitsios, and A. Tzes, "Guidance and control design for a class of spin-stabilized fin-controlled projectiles," Journal of Guidance, Control, and Dynamics, vol. 36, no. 2, pp. 517-531, 2013.

[5] E. Gagnon and M. Lauzon, "Course correction fuse concept analysis for in-service $155 \mathrm{~mm}$ spin-stabilized gunnery projectiles," in Proceedings of the AIAA Guidance, Navigation and Control Conference and Exhibit, pp. 1-20, Honolulu, Hawaii, USA, August 2008.

[6] F. Fresconi, G. Cooper, I. Celmins, J. DeSpirito, and M. Costello, "Flight mechanics of a novel guided spin-stabilized projectile concept," Journal of Aerospace Engineering, vol. 226, no. 3, pp. 327-340, 2012.

[7] M. Gross, M. Costello, and F. Fresconi, "Impact point model Predictive control of a spin-stabilized projectile with instability protection," in Proceedings of the AIAA Atmospheric Flight Mechanics Conference, pp. 1-21, Boston, Mass, USA, 2013.

[8] M. Costello and A. Peterson, "Linear theory of a dual-spin projectile in atmospheric flight," Journal of Guidance, Control, and Dynamics, vol. 23, no. 5, pp. 789-797, 2000.

[9] C. Yang, C. Lin, K. Chopper et al., "Analysis and evaluation of guidance integrated fuzing," AIAA 93-3786CP, AIAA, 1993.

[10] D. Corriveau, C. Berner, and V. Fleck, "Trajectory correction using impulse thrusters for conventional artillery projectiles," in Proceedings of the 23th International Symposium on Ballistics, pp. 639-646, Tarragona, Spain, 2007.

[11] D. Corriveau, P. Wey, and C. Berner, "Thrusters pairing guidelines for trajectory corrections of projectiles," Journal of Guidance, Control, and Dynamics, vol. 34, no. 4, pp. 1120-1128, 2011.

[12] A. Calise and H. El-Shirbiny, "An analysis of aerodynamic control for direct fire spinning projectiles," in Proceedings of the AIAA Guidance, Navigation, and Control Conference and Exhibit, pp. 1-10, Montreal, Canada, August 2001.

[13] A. Calise, H. El-Shirbiny, and N. Kim, "An adaptive guidance approach for spinning projectiles," in Proceedings of the AIAA Guidance, Navigation, and Control Conference and Exhibit, pp. 1-13, Providence, RI, USA, August 2004.

[14] G. Frost and M. Costello, "Control authority of a projectile equipped with an internal unbalanced part," Journal of Dynamic Systems, Measurement and Control, vol.128, no. 4, pp. 1005-1012, 2006.

[15] J. Rogers and M. Costello, "Control authority of a projectile equipped with a controllable internal translating mass," Journal of Guidance, Control, and Dynamics, vol. 31, no. 5, pp.1323-1333, 2008.

[16] D. Ollerenshaw and M. Costello, "On the swerve response of projectiles to control input," Journal of Guidance, Control, and Dynamics, vol. 31, no. 5, pp. 1259-1265, 2008.

[17] F. Fresconi and P. Plostins, "Control mechanism strategies for spin-stabilized projectiles," in Proceedings of the 47th AIAA Aerospace Sciences Meeting Including the New Horizon Forum and Aerospace Exposition, AIAA Paper 1-23, Orlando, Fla, USA, January 2009.

[18] F. Fresconi and M. Ilg, "Model predictive control of agile projectiles," in Proceedings of the AIAA Atmospheric Flight Mechanics Conference, pp. 1-16, Minneapolis, Minn, USA, 2012.

[19] R. Letniak and M. Costello, "A nonlinear model predictive observer for smart projectile applications," in Proceedings of the AIAA Atmospheric Flight Mechanics Conference and Exhibit, pp. 1-21, Hilton Head, SC, USA, August 2007. 
[20] N. Slegers, "Predictive control of a munition using low-speed linear theory," Journal of Guidance, Control, and Dynamics, vol. 31, no. 3, pp. 768-775, 2008.

[21] L. C. Hainz III and M. Costello, "Modified projectile linear theory for rapid trajectory prediction," Journal of Guidance, Control, and Dynamics, vol. 28, no. 5, pp. 1006-1014, 2005.

[22] G. Cooper, F. Fresconi, and M. Costello, "Practical assessment of real-time impact point estimators for smart weapons," in Proceedings of the AIAA Atmospheric Flight Mechanics Conference and Exhibit, pp. 1-17, Honolulu, Hawaii, USA, August 2008.

[23] A. K. Ghosh and O. Prakash, "Neural models for predicting trajectory performance of an artillery rocket," Journal of Aerospace Computing, Information and Communication, vol. 1, no. 2, pp. 112-115, 2004.

[24] K. A. Kramer and S. C. Stubberud, "Impact time and point predicted using a neural extended Kalman filter," in Proceedings of the International Conference on Intelligent Sensors, Sensor Networks and Information Processing Conference, pp. 199-204, IEEE, Melbourne, Australia, December 2005.

[25] J. Robinson and P. Strömbäck, "Perturbation based guidance for a generic 2D course correction fuze," in Proceedings of the AIAA Guidance, Navigation, and Control Conference, AIAA paper 126, Boston, Mass, USA, August 2013.

[26] C. H. Murphy, "Free flight motion of symmetric missiles," US Army Research Laboratory Report 1216, US Army Research Laboratory, Aberdeen Proving Ground, Md, USA, 1963. 

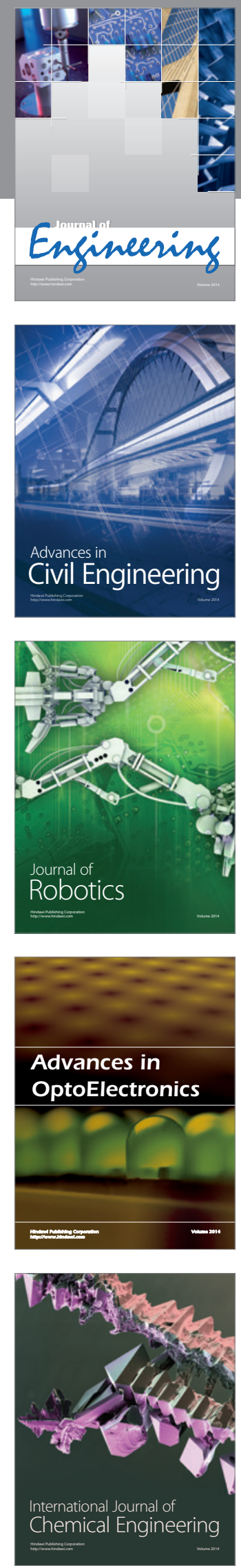

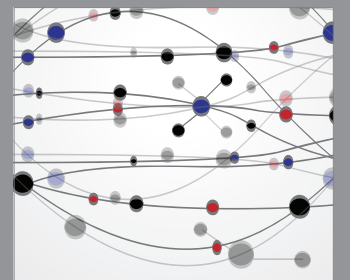

The Scientific World Journal
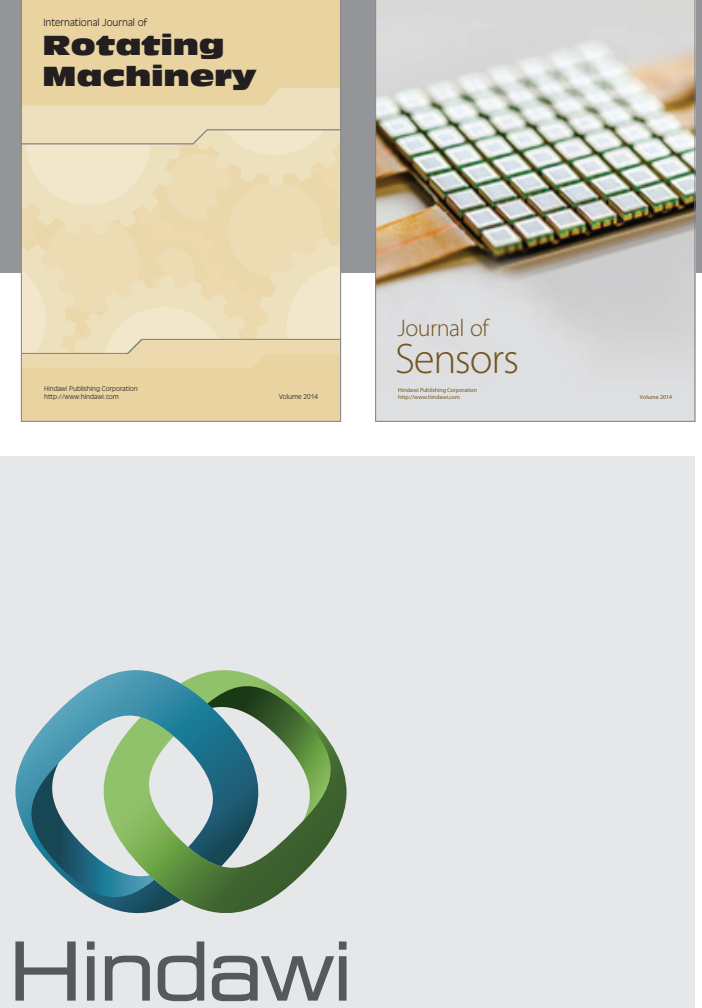

Submit your manuscripts at http://www.hindawi.com
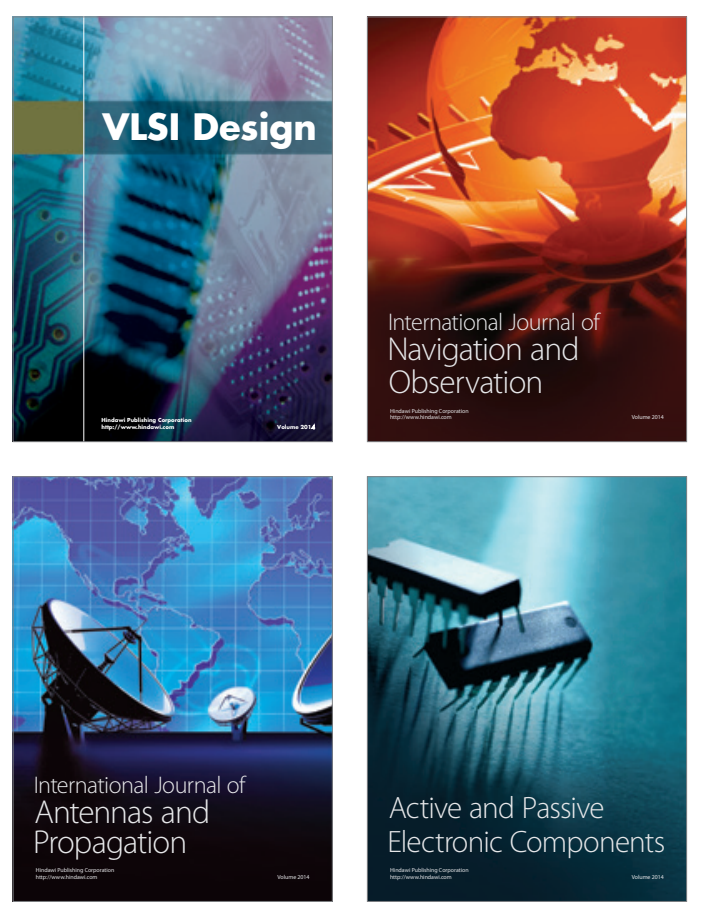
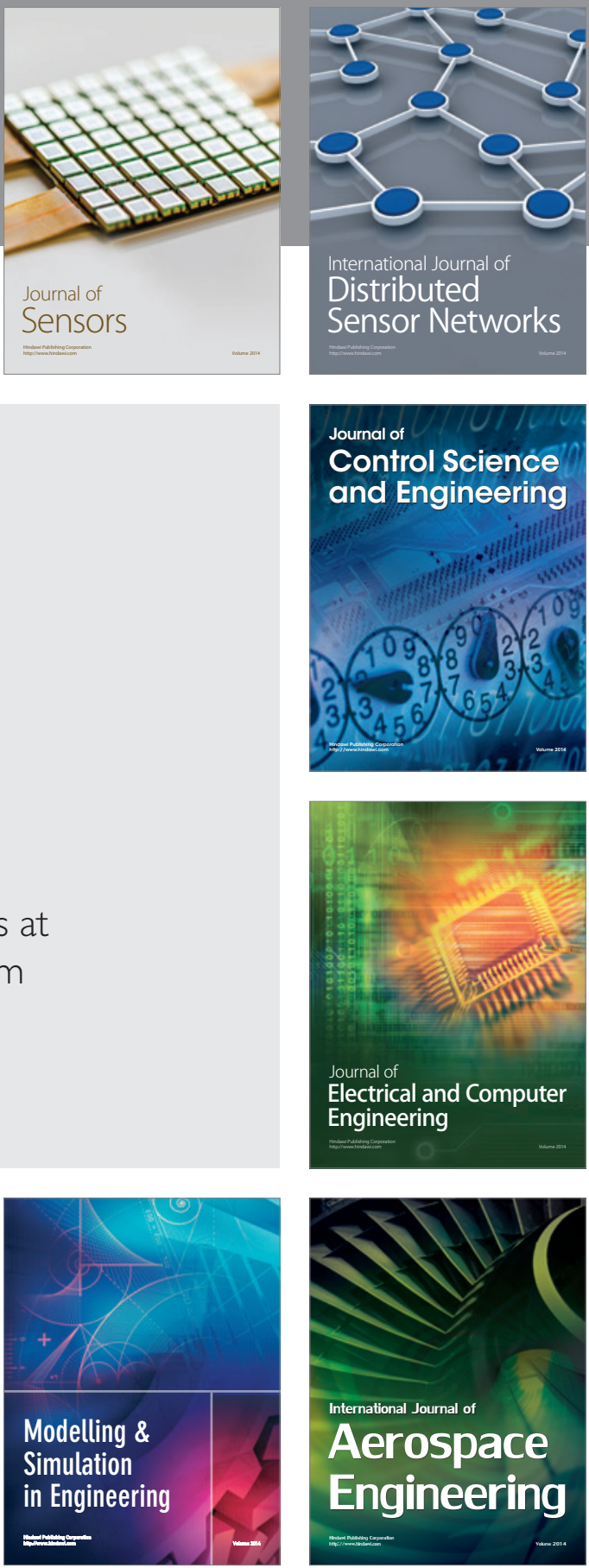

Journal of

Control Science

and Engineering
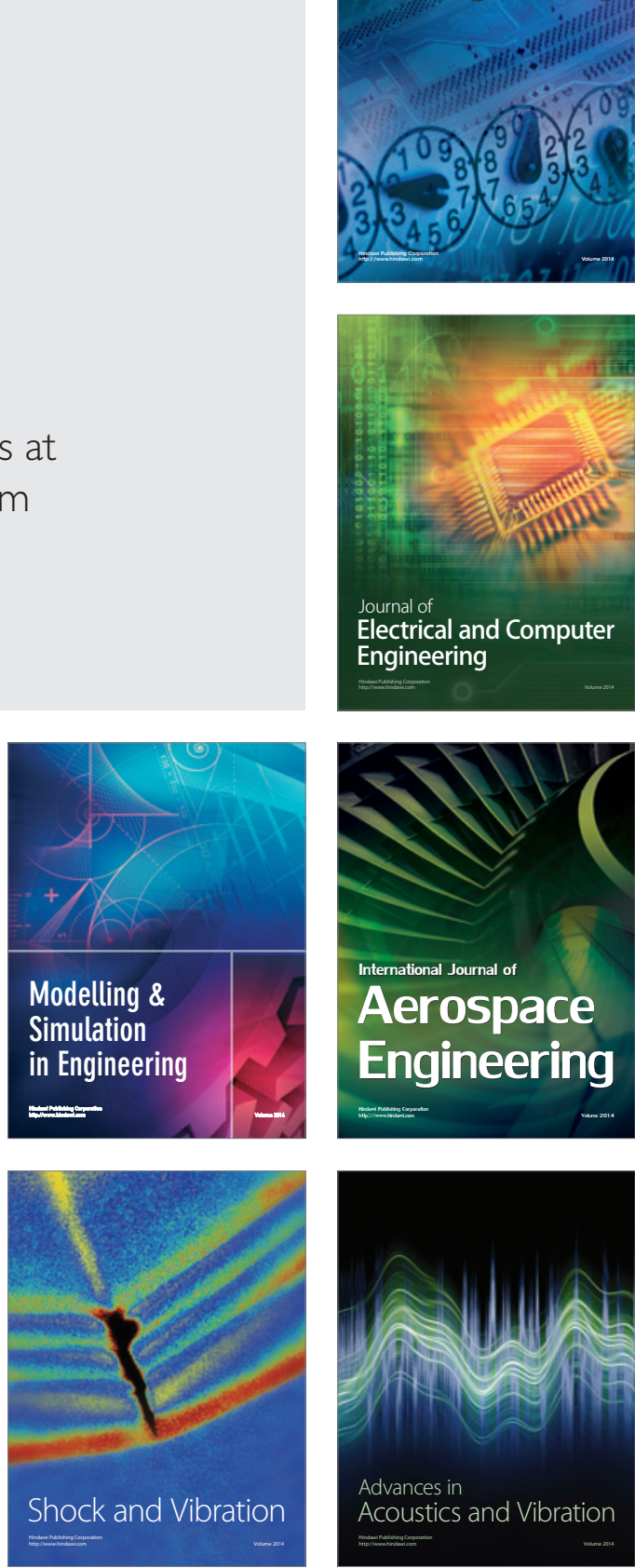